\title{
Predicting the distribution of Omalonyx (Mollusca: Pulmonata: Succineidae) species from literature review, museum databases and new sampling efforts in Brazil
}

\author{
Daniel Coscarelli, ${ }^{1,2}$ Lângia C. Montresor ${ }^{3}$, Philip Russo ${ }^{4}$, Alan Lane de Melo² \& Teofânia H.D.A. Vidigal ${ }^{* *}$ \\ ${ }^{1}$ Laboratório de Malacologia e Sistemática Molecular, Departamento de Zoologia, Universidade Federal de Minas \\ Gerais, Av. Antônio Carlos, 6627, Pampulha, Belo Horizonte, MG, Brazil. \\ ${ }^{2}$ Departamento de Parasitologia, Instituto de Ciências Biológicas, Universidade Federal de Minas Gerais, \\ Av. Antônio Carlos, 6627, Pampulha, Belo Horizonte, MG, Brazil. \\ ${ }^{3}$ Moluscário Lobato Paraense, Centro de Pesquisas René Rachou, Fiocruz, Av. Augusto de Lima, 1715, Barro Preto, \\ Belo Horizonte, MG, Brazil. \\ ${ }^{4}$ Laboratório de Aracnologia, Departamento de Zoologia, Instituto de Ciências Biológicas, Universidade Federal de \\ Minas Gerais, Av. Antonio Carlos 6627 - Pampulha, Belo Horizonte, MG, Brazil. \\ *Corresponding author: Teofânia H.D.A. Vidigal, email: teofania.vidigal@gmail.com
}

COSCARELLI, D., MONTRESOR, L. C., RUSSO, P., MELO A. L., VIDIGAL T. H. D. A. Predicting the distribution of Omalonyx (Mollusca: Pulmonata: Succineidae) from literature review, museum data bases and new sampling efforts in Brazil. Biota Neotropica. 18(1): e20170409. http://dx.doi.org/10.1590/1676-0611-BN-2017-0409

\begin{abstract}
Accurate distributional information is crucial for studies on systematics, biodiversity and conservation. To improve the knowledge regarding the geographical distribution of Omalonyx in South America, we present updated information based on data from a literature review, institutional collections and malacological surveys. All this information composed the dataset used to predict species distribution employing the Maximum Entropy Algorithm (MaxEnt). The model was run using data on species distribution, altitude and bioclimatic variables (WorldClim database). The model had consistent performance, and areas presenting similar conditions to areas where the species were recorded were considered areas of occurrence. The predicted occurrence areas included those that were already surveyed and those that are considered potential occurrence areas. The results demonstrate that the genus has widespread distribution in the Neotropical region and occurs in the tropical, temperate and arid regions of South America and Lesser Antilles. Omalonyx spp. were recorded in all South American countries and hydrographic regions. However, in some countries, there were only isolated records (ex: Colombia and Ecuador). Here, we also present the first record of Omalonyx spp. in four Brazilian States (Acre, Rondônia, Piaui, and Amapá). The genus was found in all hydrographic regions within Brazil and among 27 federative unities; it was absent from only two unities (Roraima State and Distrito Federal). This work contributes to the knowledge on Omalonyx spp. distribution and provides an important basis for the work of ecologists and taxonomists.
\end{abstract}

Keywords: MaxEnt, niche model, species distribution, Modeling, Succineids, Neotropic.

\section{Predição da distribuição de Omalonyx (Mollusca: Pulmonata: Succineidae) a partir da revisão da literatura, dados de museu e novo esforço amostral no Brasil}

Resumo: A informação precisa sobre a distribuição é crucial para os estudos sobre sistemática, biodiversidade e conservação. Para melhorar o conhecimento sobre a distribuição geográfica de Omalonyx na América do Sul, apresentamos informações atualizadas com base em dados de revisão de literatura, coleções institucionais e pesquisas malacológicas. Toda essa informação compôs o conjunto de dados usado para predição da distribuição de espécies empregando o Algoritmo de Entropia Máxima (MaxEnt). O modelo foi executado usando dados de distribuição de espécies, altitude e variáveis bioclimáticas (banco de dados WorldClim). O modelo apresentou um desempenho consistente e as áreas que apresentaram condições semelhantes às áreas onde as espécies foram registradas, foram consideradas áreas de ocorrência. As áreas de ocorrências previstas incluíram aquelas que já foram pesquisadas e aquelas que são consideradas áreas de ocorrência potencial. Os resultados demonstram que o gênero tem uma distribuição Neotropical ampla e que ocorre nas regiões tropical, temperada e árida da América do Sul e nas Pequenas Antilhas. Omalonyx spp. foram registradas em todos os países e bacias sul-americanas. No entanto, em alguns países, apenas registros isolados foram encontrados (ex: Colômbia e Equador). Aqui, também apresentamos o primeiro registro de Omalonyx spp. em quatro estados brasileiros (Acre, Rondônia, Piauí e Amapá). O gênero foi encontrado em todas as regiões hidrográficas no 
Brasil e nas 27 unidades federativas; sendo ausente em apenas duas unidades federativas (Estado de Roraima e Distrito Federal). Esse trabalho contribui para o conhecimento da distribuição das espécies de Omalonyx e fornece uma importante base para trabalhos de ecólogos e taxonomistas.

Palavras chave: MaxEnt, modelagem de nichos, distribuição de espécies, Modelagem, Succineídeos, Neotrópico.

\section{Introduction}

The genus Omalonyx d'Orbigny, 1837 presents a reduced shell and represents one of the slug-like lineages within the family Succineidae (Patterson 1971, Tillier 1981, Barker 2001). The earliest fossil records of Succineidae were from the Tertiary of Europe and, currently, most of the diversity within the family occurs in the islands of the Pacific, in the Indian subcontinent and the Americas (Barker 2001). These Neotropical slugs are terrestrial, live on aquatic macrophytes or riparian vegetation and can also be found in humid soil (Garcia et al. 2012). They can act as agricultural plagues (Olazarri 1979, Garcia et al. 2012) and are agents in the control of water-cress (Poi de Neiff et al. 1977). They can also act as intermediate hosts of an avian trematode, Leucochloridium (Lutz 1921, Travassos 1928), in the wild and of the nematodes Angiostrongylus costaricensis and A. vasorum in the laboratory (Montresor et al. 2008, Mozzer et al. 2011).

Currently, it is assumed that the genus comprises six recognized species (Tillier 1980, 1981, Martínez 1993, Arruda \& Thomé 2008a, b, Arruda et al. 2009, Coscarelli \& Vidigal 2011): Omalonyx convexus (Martens, 1868), Omalonyx unguis (d'Orbigny, 1837), Omalonyx brasiliensis (Simroth, 1896), Omalonyx matheroni (Pontiez \& Michaud, 1835), Omalonyx geayi Tillier, 1980, and Omalonyx pattersonae Tillier, 1981. However, identification of the species within this genus is greatly complicated by intraspecific morphological variation in the diagnostic characteristics such as the papillae on the internal surface of the penis, the insertion of the retractor muscle of the penis, the shape of papillae on the inner surface of the vagina and others (Tillier 1980, 1981, Martínez 1993, Arruda \& Thomé 2008a, b, Arruda et al. 2009, Coscarelli \& Vidigal 2011).

To improve taxonomic knowledge on Omalonyx species, studies encompassing morphological and molecular data are essential. However, the first step to elucidating these questions is to acquire knowledge on species geographical distribution. Accurate geographical distributional information is crucial to solve systematics and biogeographic problems and to drive conservation efforts. Species-distribution models infer potential distribution from data on observed distribution and from environmental variables related to the sites where occurrences were recorded (Bradie \& Leung 2017). These models assume that climate ultimately restricts a species' distribution and summarize it with a number of climatic variables within the known range of the species, generating a bioclimatic profile. They play an important role as first filters in assessing the potential distribution of each species, improving the success of future sampling efforts and providing initial insight into the bioclimatic tolerance of different species (Beaumont et al. 2005, Rubio \& Acosta 2011). These tools have also been applied in several conservation biology studies to identify areas with high species richness and to predict effects of climate change on species' distributions (Araújo \& Rahbek 2006, Costa et al. 2010, Vogler et al. 2013).

There are several methods to model species distribution, and they may use presence/absence, abundance or presence-only data. Usually, species records have limited coverage and consist of presence-only data (Elith et al. 2011). The Maximum Entropy Algorithm (MaxEnt) presents high performance for modeling presence-only data and is widely used to model species distribution (Bradie \& Leung 2017). Here, we use this method to improve the knowledge on the geographical distribution of Omalonyx spp., assembling literature data with specimens either collected in our surveys, from malacological collections or donated by partner laboratories. These data on species presence and the associated bioclimatic variables were used to model species distribution, to investigate the main environmental factors related to the occurrence of Omalonyx species and to detect species with overlapping distribution areas. This work improves the knowledge on these slugs' distribution, providing information to propel advances in the morphological and molecular taxonomy of these neotropical succineids.

\section{Material and Methods}

Literature data on Omalonyx was reviewed (until December 2016) in order to find records of its occurrence across the Neotropical region (see the topic literature review in the results). Institutions harboring preserved specimens were identified in the literature review and by onsite and virtual searching in institutional collections (Table 1), and information regarding the lot number and collection site was organized in two tables (Table 2 and 3 ). Donated specimens were also included in these tables (Table 2 and 3).

To investigate in detail the distribution of these slugs in Brazil, malacological surveys comprising all geographical regions (North, Northeast, Southeast, South and Central West) were conducted between 2006 and 2016 (permission from environmental agency: ICMBio/SISBIO no. 12113-3). Specimens were manually collected from wet soil and vegetation in the vicinity of freshwater systems. Voucher specimens were deposited in the malacological collection of the Laboratório de Malacologia e Sistemática Molecular (LMSM), at Universidade Federal de Minas Gerais, Minas Gerais, Brazil.

All localities from the literature review, museums, collections, donations and our own malacological surveys were listed (Table 2 and 3 ) and plotted on a map (Figure 1) using the software ArcGIS 10.4 (ArcGIS Desktop 10.4 Geostatistical Analyst, Environmental Systems Research Institute ESRI, 2016). The geographic position of the localities and the information about South American hydrography and geography were cross-checked with Google Earth, Global Gazetteer, Hydro web (a hydrologic information system of Agência Nacional de Águas - ANA, the national water resources agency of the Environment Ministry, Brazil). Information regarding the hydrography of Austral South America was obtained from Bonetto (1994).

Morphological identification of specimens collected in our malacological surveys was based on characteristics of the reproductive system according to the following literature: O. convexus (Hylton-Scott \& Lapuente 1968, Hylton-Scott 1971, Tillier 1981, Arruda \& Thomé 2008b), O. unguis (Coscarelli \& Vidigal 2011), O. matheroni, O. pattersonae and O. geayi (Tillier 1981). Specific identification provided in the papers (literature review) and on the labels (museum specimens) was also used in this work. Some of these records were not identified to species level and are designated Omalonyx sp. Until 1981, there were several species of Omalonyx, now recognized as synonyms. Throughout the text, species are designated according to Tillier (1981) and Arruda \& Thomé (2008a, b), where synonyms for the six valid Omalonyx species can be found. However, when consulting the topic references, the reader will find the former designation.

Data of occurrence (Table 2 and 3) were used to model the species geographic distributions using one topographic variable (altitude) and the nineteen bioclimatic variables derived from the WorldClim database v. 1.4 as showed by Vogler et al. (2013) (Table 4). The classifications of climate were based on Koppen-Geiger climate classification types for South America (Peel et al. 2007). Maps for the distribution of O. matheroni, $O$. pattersonae, O. geayi, O. convexus and $O$. unguis were produced. 
Table 1. Institutions harbouring specimens of Omalonyx found after literature review, visits and virtual search in collections databases.

\begin{tabular}{|c|c|}
\hline Abbreviation & Museum or collection name \\
\hline ANSP & Academy of Natural Sciences of Philadelphia, Pennsylvania, United States of America. \\
\hline BMNH & British Museum of Natural History, London, United Kingdom. \\
\hline CM & Carnegie Museum, Pittsburgh, Pennsylvania, United States of America. \\
\hline CMIOC $^{*}$ & Coleção de Moluscos do Instituto Oswaldo Cruz, Rio de Janeiro, Brazil. \\
\hline FLMNH & Florida Museum of Natural, United States of America. \\
\hline FMNH & Field Museum of Natural History, Chicago, Illinois, United States of America. \\
\hline INPA $^{*}$ & Instituto Nacional de Pesquisas da Amazônia, Manaus, Amazonas, Brazil. \\
\hline LMSM & Laboratório de Malacologia e Sistemática Molecular, Belo Horizonte, Minas Gerais, Brazil. \\
\hline MACN & Museo Argentino de Ciencias Naturales, Bernadino Rivadavia, Buenos Aires, Argentina. \\
\hline MCP & Museu de Ciências e Tecnologia da Pontifícia Universidade Católica do Rio Grande do Sul, Porto Alegre, Rio Grande do Sul, Brazil. \\
\hline MCNZ & Museu de Ciências Naturais, Fundação Zoobotânica do Rio Grande do Sul, Porto Alegre, Rio Grande do Sul, Brazil. \\
\hline MCZ & Museum of Comparative Zoology, Harvard University, Cambridge, Massachusetts, United States of America. \\
\hline MIRR $^{*}$ & Museu Integrado de Roraima, Boa Vista; Roraima, Brazil. \\
\hline MLP $^{*}$ & Museo de La Plata, La Plata, Argentina. \\
\hline MNHCL & Museo Nacional de Historia Natural del Chile, Chile. \\
\hline MHNG & Muséum d'Histoire Naturelle, Genève, Switzerland. \\
\hline MNKMO & Museo de Historia Natural Noel Kempff Mercado, Santa Cruz de la Sierra, Bolivia. \\
\hline MNHN & Muséum national d'Histoire naturelle, Paris, France. \\
\hline MNHNM & Museo Nacional de Historia Natural de Montevideo, Uruguay. \\
\hline MNRJ $^{*}$ & Museu Nacional da Universidade Federal do Rio de Janeiro, Rio de Janeiro, Brazil. \\
\hline MPEG $^{*}$ & Museu Paraense Emílio Goeldi, Belém, Pará, Brazil. \\
\hline MZUM & Museum of Zoology, University of Michigan, Ann Arbor, Michigan, United States of America. \\
\hline MZUSP* $^{*}$ & Museu de Zoologia da USP, São Paulo, São Paulo, Brazil. \\
\hline RMNH & Rijksmuseum van Natuurlijke Histoire, Leiden, Netherlands \\
\hline SMNH & Naturhistoriska Riksmuseet, Stockholm, Sweden. \\
\hline UF & Florida Museum of Natural History (USA). \\
\hline UFS & Universidade Federal de Sergipe, Aracajú, Sergipe, Brazil. \\
\hline USDA & United States Department of Agriculture, Philadelphia, United States of America. \\
\hline USMN & National Museum of Natural History, Smithsonian Institution, Washington, DC, United States of America. \\
\hline ZMB & Museum für Naturkunde der Humboldt Universität, Berlin, Germany. \\
\hline ZUECGAS & Museu de Zoologia da Universidade Estadual de Campinas, Campinas, São Paulo, Brazil. \\
\hline
\end{tabular}

Visited collections*

Table 2. Records of Omalonyx in Brazil based on literature data, institutional collections, and our own malacological surveys.

\begin{tabular}{|c|c|c|c|c|c|c|}
\hline Species & Region & Locality & Record & ${ }^{1}$ Source & $\begin{array}{c}{ }^{2} \text { Hydrographic } \\
\text { Region }\end{array}$ & Coordinates \\
\hline O. matheroni & North & Rio Branco, Acre & LMSM 3346, 3378-83, 3438 & Survey & 5 & $10^{\circ} 31^{\prime} 48^{\prime \prime} \mathrm{S}, 68^{\circ} 18^{\prime} 54^{\prime \prime} \mathrm{W}$ \\
\hline O. matheroni & North & $\begin{array}{c}\text { Nações Unidas Ave. Macapá, } \\
\text { Amapá }\end{array}$ & $\begin{array}{c}\text { LMSM 3205-07, 3216-17, 3144, } \\
3146\end{array}$ & Survey & 4 & $0^{\circ} 28^{\prime} 19.2^{\prime \prime} \mathrm{N}, 51^{\circ} 39^{\prime} 0^{\prime \prime} \mathrm{W}$ \\
\hline O. matheroni & North & Amazon River, Santana, Amapá & LMSM 3199, 3200-03, 3232-33 & Survey & 4 & $0^{\circ} 33^{\prime} 50.4^{\prime \prime} \mathrm{N}, 51^{\circ} \mathrm{W} 10^{\prime} 35.04^{\prime \prime}$ \\
\hline O. matheroni & North & BR 156 Road, Calçoene, Amapá & $\begin{array}{c}\text { LMSM } 3215,3220-21,3145 \\
3147\end{array}$ & Survey & 4 & $2^{\circ} 29^{\prime} 44.16^{\prime \prime} \mathrm{N}, 50^{\circ} 57^{\prime} 14.04 \mathrm{~W}^{\prime}$ \\
\hline Omalonyx sp. & North & Manicore, Amazonas & INPA 445 & Museum & 4 & $6^{\circ} 27^{\prime} 35.28^{\prime \prime} \mathrm{S}, 61^{\circ} 26^{\prime} 47.4 \mathrm{~W}$ " \\
\hline Omalonyx sp. & North & Novo Aripuanã, Amazonas & $\begin{array}{c}\text { INPA769, 779, 781, 785, 801, 802, } \\
808 \text { (Pimpão 2007) }\end{array}$ & Literature & 5 & $6^{\circ} 49^{\prime} 30^{\prime \prime} \mathrm{S}, 60^{\circ} 28^{\prime} 37.92^{\prime \prime} \mathrm{W}$ \\
\hline Omalonyx sp. & North & Juruá River, Amazonas & MZSP 18444,31962 & Museum & 5 & $2^{\circ} 44^{\prime} 19.68^{\prime \prime} \mathrm{S}, 66^{\circ} 45^{\prime} 41.76^{\prime \prime} \mathrm{W}$ \\
\hline Omalonyx sp. & North & Barurua Island, Amazonas & MZSP 18445 & Museum & 5 & $2^{\circ} 31^{\prime} 48^{\prime \prime} \mathrm{S}, 67^{\circ} 21^{\prime} 59.76^{\prime \prime} \mathrm{W}$ \\
\hline Omalonyx sp. & North & $\begin{array}{c}\text { Amaña Lake, Japurá River } \\
\text { Basin, Amazonas }\end{array}$ & MZSP 31874 & Museum & 5 & $2^{\circ} 35^{\prime} 53.16^{\prime \prime} \mathrm{S}, 64^{\circ} 39^{\prime} 47.16^{\prime \prime} \mathrm{W}$ \\
\hline Omalonyx sp. & North & Boca do Acre, Amazonas & MZSP 31881-83 & Museum & 5 & $8^{\circ} 45^{\prime} 37.44^{\prime \prime} \mathrm{S}, 68^{\circ} 53^{\prime} 52.8^{\prime \prime} \mathrm{W}$ \\
\hline Omalonyx sp. & North & Manaus, Amazonas & $\begin{array}{c}\text { INPA 575, 598, 614, 620, 858, } \\
\text { 1081; MZSP } 31963\end{array}$ & Museum & 5 & $3^{\circ} 6^{\prime} 6.84^{\prime \prime} \mathrm{S}, 60^{\circ} 15^{\prime} 0^{\prime \prime} \mathrm{W}$ \\
\hline O. pattersonae & North & Itacotiara, Manaus, Amazonas & ANSP 109524 & Museum & 5 & \\
\hline \multirow[t]{2}{*}{ Omalonyx sp. } & North & Marchantaria Island, Amazonas & INPA 522 & Museum & 5 & $3^{\circ} 14^{\prime} 23.28^{\prime \prime} \mathrm{S}, 59^{\circ} 56^{\prime} 15.36^{\prime \prime} \mathrm{W}$ \\
\hline & North & & & & & \\
\hline O. geayi & North & $\begin{array}{c}\text { Careiro Marchantaria Island, } \\
\text { Amazonas }\end{array}$ & MCNZ 30548 (Arruda et al. 2016) & Literature & 5 & $3^{\circ} 15^{\prime} 14.11^{\prime \prime} \mathrm{S}, 59^{\circ} 58^{\prime} 19.09^{\prime \prime} \mathrm{W}$ \\
\hline Omalonyx sp. & North & Parana Uauaçu, Amazonas & INPA 532 & Museum & 5 & $4^{\circ} 18^{\prime} 50.04^{\prime \prime} \mathrm{S}, 62^{\circ} 9^{\prime} 55.08^{\prime \prime} \mathrm{W}$ \\
\hline
\end{tabular}


Coscarelli, D. et al.

Table 2. Continued..

\begin{tabular}{|c|c|c|c|c|c|c|}
\hline Species & Region & Locality & Record & ${ }^{1}$ Source & $\begin{array}{c}{ }^{2} \text { Hydrographic } \\
\text { Region }\end{array}$ & Coordinates \\
\hline O.matheroni & North & Iranduba, Amazonas & LMSM 2270, 2276-77 & Survey & & $3^{\circ} 57^{\prime} 7.2^{\prime \prime} \mathrm{S}, 60^{\circ} 28^{\prime} 50.52^{\prime \prime} \mathrm{W}$ \\
\hline O.matheroni & North & Iranduba, Amazonas & INPA 551-2, 870 & Museum & 5 & $3^{\circ} 57^{\prime} 7.2^{\prime \prime} \mathrm{S}, 60^{\circ} 28^{\prime} 50.52^{\prime \prime} \mathrm{W}$ \\
\hline O. geayi & North & Iranduba, Amazonas & INPA 1091 (Arruda et al. 2016) & Literature & 5 & $\begin{array}{c}3^{\circ} 8^{\prime} 44.4768^{\prime \prime} \mathrm{S}, 60^{\circ} \\
155^{\prime} 26.6256^{\prime} \mathrm{W}\end{array}$ \\
\hline O. matheroni & North & Preto da Eva River, Amazonas & LMSM 2260, 2332-35 & Survey & 5 & $2^{\circ} 31^{\prime} 47.64^{\prime \prime} \mathrm{S}, 59^{\circ} 38^{\prime} 17.52^{\prime \prime} \mathrm{W}$ \\
\hline O. matheroni & North & Preto da Eva River, Amazonas & INPA 1090 (Garcia et al. 2012) & Literature & 5 & $2^{\circ} 31^{\prime} 47.64^{\prime \prime} \mathrm{S}, 59^{\circ} 38^{\prime} 17.52^{\prime \prime} \mathrm{W}$ \\
\hline Omalonyx sp. & North & Preto da Eva River, Amazonas & INPA 1090 (Garcia et al. 2012) & Literature & 5 & $2^{\circ} 31^{\prime} 47.64^{\prime \prime} \mathrm{S}, 59^{\circ} 38^{\prime} 17.52^{\prime \prime} \mathrm{W}$ \\
\hline O. pattersonae & North & Preto da Eva River, Amazonas & LMSM 2260, 2332-35; & Survey & 5 & $2^{\circ} 31^{\prime} 47.64^{\prime \prime} \mathrm{S}, 59^{\circ} 38^{\prime} 17.52^{\prime \prime} \mathrm{W}$ \\
\hline O. pattersonae & North & Preto da Eva River, Amazonas & INPA 1090 (Garcia et al. 2012) & Literature & 5 & $2^{\circ} 31^{\prime} 47.64^{\prime \prime} \mathrm{S}, 59^{\circ} 38^{\prime} 17.52^{\prime \prime} \mathrm{W}$ \\
\hline Omalonyx sp. & North & Carauari, Amazonas & MCP 09184 & Museum & 5 & $5^{\circ} 7^{\prime} 7.68^{\prime \prime} \mathrm{S}, 67^{\circ} 20^{\prime} 15.36^{\prime \prime} \mathrm{W}$ \\
\hline O. geayi & North & Carauari, Amazonas & $\begin{array}{l}\text { INPA 1643; MCP 09183 } \\
\text { (Arruda et al. 2016) }\end{array}$ & Literature & 5 & $5^{\circ} 7^{\prime} 7.68^{\prime \prime S}, 67^{\circ} 20^{\prime} 15.36^{\prime \prime} \mathrm{W}$ \\
\hline O. matheroni & North & & SMNH 1450 (Tillier 1981) & Literature & & unespecified locality \\
\hline O. matheroni & North & - & MHNG 53834.8 (Tillier 1981) & Literature & & unespecified locality \\
\hline Omalonyx sp. & North & Santarém, Pará & $\begin{array}{c}\text { ZUECGAS 3313, 3215-3217; } \\
\text { MZSP 31885 } \\
\end{array}$ & Museum & 5 & $2^{\circ} \mathrm{S} 45^{\prime} 52.56^{\prime \prime}, 55^{\circ} \mathrm{W} 56^{\prime} 16.8^{\prime \prime}$ \\
\hline O. matheroni & North & Santarém, Pará & $\begin{array}{c}\text { LMSM } 2573-2579,2583,2646- \\
49,2663,2670,2761,2765-67 \\
2789-94,2835,2849-76,2910 \\
2930,3234-36\end{array}$ & Survey & 5 & $2^{\circ} 45^{\prime} 52.56^{\prime \prime} \mathrm{S}, 55^{\circ} 56^{\prime} 16.8^{\prime \prime} \mathrm{W}$ \\
\hline O. matheroni & North & Santarém, Pará & $\begin{array}{c}\text { Lange de Morretes 1949; } \\
\text { BMNH 1896.9.33 (Tillier 1981), } \\
\text { Simone 2006 } \\
\end{array}$ & Literature & 5 & $2^{\circ} 45^{\prime} 52.56^{\prime} \mathrm{S}, 55^{\circ} 56^{\prime} 16.8^{\prime \prime} \mathrm{W}$ \\
\hline O. matheroni & North & Alenquer, Pará & FMNH 29190 (Tillier 1981) & Literature & 5 & $0^{\circ} 32^{\prime} 10.68^{\prime \prime} \mathrm{S}, 55^{\circ} 41^{\prime} 56.4^{\prime \prime} \mathrm{W}$ \\
\hline Omalonyx sp. & North & Almerim, Pará & MZSP 31877 & Museum & 5 & $0^{\circ} 17^{\prime} 56.4^{\prime \prime} \mathrm{N}, 53^{\circ} 53^{\prime} 37.68^{\prime \prime} \mathrm{W}$ \\
\hline Omalonyx sp. & North & Furo do Jurupari, Pará & MZSP 31879 & Museum & 5 & $2^{\circ} 40^{\prime} 37.2^{\prime \prime} \mathrm{S}, 53^{\circ} 16^{\prime} 48^{\prime \prime} \mathrm{W}$ \\
\hline Omalonyx sp. & North & Lago Parú, Pará & MZSP 31959 & Museum & 5 & $1^{\circ} 53^{\prime} 30.12^{\prime \prime} \mathrm{S}, 55^{\circ} 49^{\prime} 55.92^{\prime \prime} \mathrm{W}$ \\
\hline O. matheroni & North & Tucuruí, Pará & $\begin{array}{c}\text { LMSM } 2569,2584-87,2588, \\
2672,2915-16\end{array}$ & Survey & 06/jul & $3^{\circ} 51^{\prime} 20.88^{\prime \prime} \mathrm{S}, 49^{\circ} 49^{\prime} 14.88^{\prime \prime} \mathrm{W}$ \\
\hline O. matheroni & North & Juruti, Pará & LMSM $3151-52,3168$ & Survey & 5 & $2^{\circ} 37^{\prime} 21.72^{\prime \prime S}, 56^{\circ} 13^{\prime} 16.32^{\prime \prime} \mathrm{W}$ \\
\hline O. matheroni & North & Belém, Pará & LMSM 3230-31 & Survey & 7 & $1^{\circ} 14^{\prime} 26.52^{\prime \prime} \mathrm{S}, 48^{\circ} 27^{\prime} 33.84^{\prime \prime} \mathrm{W}$ \\
\hline O. matheroni & North & Cereja River, Bragança, Pará & LMSM 4037-4043 & Survey & 7 & $1^{\circ} 20^{\prime} 49.2^{\prime \prime} \mathrm{S}, 46^{\circ} 45^{\prime} 42.84^{\prime \prime} \mathrm{W}$ \\
\hline O. pattersonae & North & Cereja River, Bragança, Pará & LMSM 4037-4043 & Survey & 7 & $1^{\circ} 20^{\prime} 49.2^{\prime \prime} \mathrm{S}, 46^{\circ} 45^{\prime} 42.84^{\prime \prime} \mathrm{W}$ \\
\hline Omalonyx sp. & North & Curuá, Pará & ZUECGAS 3204-9, 3210-4, 3218 & Museum & 7 & $1^{\circ} 50^{\prime} 45.24^{\prime \prime} \mathrm{S}, 55^{\circ} 6^{\prime} 47.52^{\prime \prime} \mathrm{W}$ \\
\hline O. matheroni & North & Ji-Paraná, Rondônia & LMSM3360, 3362, 3367 & Survey & 5 & $10^{\circ} 27^{\prime} 43.92^{\prime \prime} \mathrm{S}, 61^{\circ} 45^{\prime} 25.56^{\prime \prime} \mathrm{W}$ \\
\hline O. matheroni & North & $\begin{array}{c}\text { Madeira River, Porto Velho, } \\
\text { Rondônia }\end{array}$ & $\begin{array}{c}\text { LMSM } 3347-48,3358-59,3364- \\
65,3372-76,3403-04,3440\end{array}$ & Survey & 5 & $8^{\circ} 46^{\prime} 14.16^{\prime \prime} \mathrm{S}, 63^{\circ} 54^{\prime} 29.16^{\prime \prime} \mathrm{W}$ \\
\hline Omalonyx sp. & Northeast & $\begin{array}{c}\text { Malvina District, Arari, } \\
\text { Maranhão }\end{array}$ & $\begin{array}{c}\text { MNRJ 6305; CMIOC } 9519 \\
\text { (Cantanhede et al. 2014) }\end{array}$ & Museum & 7 & $3^{\circ} 27^{\prime} 30.96^{\prime \prime} \mathrm{S}, 44^{\circ} 46^{\prime} 30^{\prime \prime} \mathrm{W}$ \\
\hline Omalonyx sp. & Northeast & $\begin{array}{c}\text { Amaral Village, Monção, } \\
\text { Maranhão }\end{array}$ & $\begin{array}{c}\text { CMIOC 9409 } \\
\text { (Cantanhede et al. 2014) } \\
\end{array}$ & Literature & 7 & $3^{\circ} 30^{\prime} 7.92^{\prime \prime S}, 45^{\circ} 16^{\prime} 33.6^{\prime \prime} \mathrm{W}$ \\
\hline Omalonyx sp. & Northeast & Palmeirândia, Maranhão & Cantanhede et al. 2014 & Literature & 7 & $2^{\circ} 41^{\prime} 34.08^{\prime \prime} \mathrm{S}, 45^{\circ} 15^{\prime} 0^{\prime \prime} \mathrm{W}$ \\
\hline Omalonyx sp. & Northeast & Pedro do Rosário, Maranhão & Cantanhede et al. 2014 & Literature & 7 & $2^{\circ} 59^{\prime} 20.76^{\prime} \mathrm{S}, 45^{\circ} 25^{\prime} 48^{\prime \prime} \mathrm{W}$ \\
\hline Omalonyx sp. & Northeast & Peri-Mirim, Maranhão & Cantanhede et al. 2014 & Literature & 7 & $2^{\circ} 34^{\prime} 18.12^{\prime \prime} \mathrm{S}, 44^{\circ} 53^{\prime} 43.44^{\prime \prime} \mathrm{W}$ \\
\hline Omalonyx sp. & Northeast & $\begin{array}{c}\text { Santa Terezinha District, } \\
\text { Pinheiro, Maranhão } \\
\end{array}$ & $\begin{array}{c}\text { CMIOC 9452-53 } \\
\text { (Cantanhede et al. 2014) }\end{array}$ & Literature & 7 & $2^{\circ} 30^{\prime} 55.08^{\prime \prime} \mathrm{S}, 45^{\circ} 48^{\prime} 7.2^{\prime \prime} \mathrm{W}$ \\
\hline Omalonyx sp. & Northeast & Santa Helena, Maranhão & Cantanhede et al. 2014 & Literature & 7 & $2^{\circ} 25^{\prime} 33.96^{\prime \prime} \mathrm{S}, 45^{\circ} 22^{\prime} 59.52^{\prime \prime} \mathrm{W}$ \\
\hline Omalonyx sp. & Northeast & $\begin{array}{l}\text { Porto Grande District, São } \\
\text { Bento, Maranhão }\end{array}$ & $\begin{array}{c}\text { CMIOC } 9471 \\
\text { (Cantanhede et al. 2014) }\end{array}$ & Literature & 7 & $2^{\circ} 42^{\prime} 0^{\prime \prime} \mathrm{S}, 44^{\circ} 49^{\prime} 21.36^{\prime \prime} \mathrm{W}$ \\
\hline Omalonyx sp. & Northeast & São João Batista, Maranhão & Cantanhede et al. 2014 & Literature & 7 & $3^{\circ} 27^{\prime} 36^{\prime \prime} \mathrm{S}, 44^{\circ} 45^{\prime} 32.04^{\prime \prime} \mathrm{W}$ \\
\hline Omalonyx sp. & Northeast & Vitória do Mearim, Maranhão & Cantanhede et al. 2014 & Literature & 7 & $3^{\circ} 34^{\prime} 26.4^{\prime \prime} \mathrm{S}, 44^{\circ} 55^{\prime} 24.96^{\prime \prime} \mathrm{W}$ \\
\hline Omalonyx sp. & Northeast & Parnaíba, Piauí & LMSM 4558-64 & Survey & 7 & $2^{\circ} 57^{\prime} 37.8^{\prime \prime} \mathrm{S}, 41^{\circ} 45^{\prime} 12.96^{\prime \prime} \mathrm{W}$ \\
\hline O. unguis & Northeast & Baturité, Ceará & MNRJ 12382 & Museum & 7 & $4^{\circ} 22^{\prime} 49.44^{\prime \prime} \mathrm{S}, 38^{\circ} 51^{\prime} 10.08^{\prime \prime} \mathrm{W}$ \\
\hline O. matheroni & Northeast & Fortaleza, Ceará & LMSM 2027-29, 4579-4580 & Survey & 7 & $3^{\circ} 47^{\prime} 10.32^{\prime \prime} \mathrm{S}, 38^{\circ} 31^{\prime} 38.64^{\prime \prime} \mathrm{W}$ \\
\hline Omalonyx sp. & Northeast & Fortaleza, Ceará & LMSM 2027-29, 4579-4580 & Survey & 7 & $3^{\circ} 47^{\prime} 10.32^{\prime \prime} \mathrm{S}, 38^{\circ} 31^{\prime} 38.64^{\prime \prime} \mathrm{W}$ \\
\hline Omalonyx sp. & Northeast & Redenção, Ceará & CMIOC 5155 & Museum & 7 & $4^{\circ} 14^{\prime} 57.48^{\prime \prime} \mathrm{S}, 38^{\circ} 45^{\prime} 56.52^{\prime \prime} \mathrm{W}$ \\
\hline Omalonyx sp. & Northeast & Sobral, Ceará & LMSM 4581-2 & Survey & 7 & $3^{\circ} 42^{\prime} 54^{\prime \prime} \mathrm{S}, 40^{\circ} 20^{\prime} 58.92^{\prime \prime} \mathrm{W}$ \\
\hline O. matheroni & Northeast & Natal, Rio Grande do Norte & $\begin{array}{c}\text { LMSM } 2491,2514,2537, \\
2540-41,3243\end{array}$ & Survey & 7 & $5^{\circ} 48^{\prime} 11.88^{\prime \prime} \mathrm{S}, 35^{\circ} 13^{\prime} 44.4^{\prime \prime} \mathrm{W}$ \\
\hline
\end{tabular}


Table 2. Continued...

\begin{tabular}{|c|c|c|c|c|c|c|}
\hline Species & Region & Locality & Record & ${ }^{1}$ Source & $\begin{array}{c}{ }^{2} \text { Hydrographic } \\
\text { Region }\end{array}$ & Coordinates \\
\hline O. pattersonae & Northeast & Natal, Rio Grande do Norte & $\begin{array}{c}\text { LMSM } 2491,2514,2537, \\
2540-41,3243\end{array}$ & Survey & 7 & $5^{\circ} 48^{\prime} 11.88^{\prime \prime} \mathrm{S}, 35^{\circ} 13^{\prime} 44.4^{\prime \prime} \mathrm{W}$ \\
\hline O. matheroni & Northeast & $\begin{array}{c}\text { Formosa Bay, } \\
\text { Rio Grande do Norte }\end{array}$ & CMIOC 8569 & Museum & 7 & $6^{\circ} 22^{\prime} 15.24^{\prime \prime} \mathrm{S}, 35^{\circ} 30^{\prime} 0^{\prime \prime} \mathrm{W}$ \\
\hline Omalonyx sp. & Northeast & João Pessoa, Paraíba & MZSP 41365 & Museum & 7 & $7^{\circ} 9^{\prime} 59.04^{\prime \prime} \mathrm{S}, 34^{\circ} 52^{\prime} 10.92^{\prime \prime} \mathrm{W}$ \\
\hline O. matheroni & Northeast & Caruaru, Pernambuco & LMSM 2783, 2837, 2909 & Survey & 7 & $8^{\circ} 10^{\prime} 48^{\prime} \mathrm{S}, 36^{\circ} 9^{\prime} 54^{\prime \prime} \mathrm{W}$ \\
\hline O. matheroni & Northeast & Caruaru, Pernambuco & $\begin{array}{l}\text { ANSPA18032-A18038 } \\
\text { (Dutra-Clarke et al. 2001) }\end{array}$ & Literature & 7 & $8^{\circ} 10^{\prime} 48^{\prime \prime} \mathrm{S}, 36^{\circ} 9^{\prime} 54^{\prime \prime} \mathrm{W}$ \\
\hline O. matheroni & Northeast & Pernambuco & BMNH 1887.9.8.31 (Tillier 1981) & Literature & 8 & $8^{\circ}$ 28'56.64”S, 37 46’21.72”W \\
\hline Omalonyx sp. & Northeast & Tacaratu, Pernambuco & MZSP 31960 & Museum & 7 & 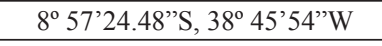 \\
\hline Omalonyx sp. & Northeast & $\begin{array}{c}\text { Dois Irmãos Zoo, Recife, } \\
\text { Pernambuco }\end{array}$ & MZSP 31967, 47767-818, 48050 & Museum & 9 & $8^{\circ} 6^{\prime} 0^{\prime \prime} \mathrm{S}, 34^{\circ} 56^{\prime} 51.36^{\prime \prime} \mathrm{W}$ \\
\hline Omalonyx sp. & Northeast & Maceió, Alagoas & MNRJ 32140 & Museum & 9 & $9^{\circ} 31^{\prime} 12.36^{\prime \prime} \mathrm{S}, 35^{\circ} 42^{\prime} 37.08^{\prime \prime} \mathrm{W}$ \\
\hline O. geayi & Northeast & Satuba, Alagoas & ZMB 90832 (Arruda et. al. 2016) & Literature & 7 & $\begin{array}{c}9^{\circ} 34^{\prime} 23.1924^{\prime} \mathrm{S}, 35^{\circ} \\
49^{\prime} 31.8216^{\prime \prime} \mathrm{W} \\
\end{array}$ \\
\hline O. pattersonae & Northeast & Itabaiana, Sergipe & MCP 09276 & Literature & 9 & $10^{\circ} 41^{\prime} 9.24^{\prime \prime S}, 37^{\circ} 25^{\prime} 18.84^{\prime \prime} \mathrm{W}$ \\
\hline O. convexus & Northeast & Itabaiana, Sergipe & $\begin{array}{c}\text { UFS 400, } 403 \text { (Jesus \& Manso } \\
\text { 2010) }\end{array}$ & Literature & 9 & $10^{\circ} 41^{\prime} 9.24^{\prime \prime} \mathrm{S}, 37^{\circ} 25^{\prime} 18.84^{\prime \prime} \mathrm{W}$ \\
\hline O. matheroni & Northeast & Tororó Dam, Salvador, Bahia & LMSM $3261-62$ & Survey & 9 & $12^{\circ} 59^{\prime} 42^{\prime \prime S}, 38^{\circ} 30^{\prime} 22.32^{\prime \prime} \mathrm{W}$ \\
\hline Omalonyx sp. & Northeast & Tororó Dam, Salvador, Bahia & CMIOC 9865;MNRJ50523 & Museum & 9 & $12^{\circ} 59^{\prime} 42^{\prime \prime S}, 38^{\circ} 30^{\prime} 22.32^{\prime \prime} \mathrm{W}$ \\
\hline O. unguis & Northeast & Tororó Dam, Salvador, Bahia & $\begin{array}{c}\text { Moricand 1836; } \\
\text { Hidalgo 1870, } 1872 \\
\end{array}$ & Literature & 9 & $12^{\circ} 59^{\prime} 42^{\prime \prime} \mathrm{S}, 38^{\circ} 30^{\prime} 22.32^{\prime \prime} \mathrm{W}$ \\
\hline O. matheroni & Northeast & Vila Nova, Bahia & Lange de Morretes 1949 & Literature & 9 & $17^{\circ} 30^{\prime} 14.04^{\prime \prime} \mathrm{S}, 40^{\circ} 30^{\prime} 28.8^{\prime \prime} \mathrm{W}$ \\
\hline Omalonyx sp. & Northeast & Uruçuca, Bahia & MZSP 18443 & Museum & 9 & $14^{\circ} 30^{\prime} 58.32^{\prime \prime} \mathrm{S}, 39^{\circ} 13^{\prime} 35.76^{\prime \prime} \mathrm{W}$ \\
\hline Omalonyx sp. & Northeast & Campo Formoso, Bahia & MZSP 31880 & Museum & 8 & $10^{\circ} 15^{\prime} 39.6^{\prime} \mathrm{S}, 40^{\circ} 42^{\prime} 33.84^{\prime \prime} \mathrm{W}$ \\
\hline O. matheroni & Northeast & Prado, Bahia & LMSM 2914 & Survey & 9 & $17^{\circ} 7^{\prime} 55.2^{\prime \prime} \mathrm{S}, 39^{\circ} 21^{\prime} 7.56^{\prime \prime} \mathrm{W}$ \\
\hline Omalonyx sp. & Northeast & Esplanada, Bahia & CMIOC 7973 & Museum & 9 & $11^{\circ} 56^{\prime} 23.64^{\prime \prime} \mathrm{S}, 37^{\circ} 53^{\prime} 10.68^{\prime \prime} \mathrm{W}$ \\
\hline Omalonyx sp. & Northeast & Entre Rios, Bahia & CMIOC 8083 & Museum & 9 & $12^{\circ} 43^{\prime} 26.4^{\prime \prime} \mathrm{S}, 38^{\circ} 25^{\prime} 22.8^{\prime \prime} \mathrm{W}$ \\
\hline Omalonyx sp. & Northeast & Wenceslau Guimarães, Bahia & CMIOC 8803 & Museum & 9 & $13^{\circ} 37^{\prime} 49.44^{\prime \prime} \mathrm{S}, 39^{\circ} 37^{\prime} 36.48^{\prime \prime} \mathrm{W}$ \\
\hline O. matheroni & Southeast & Maracás, Bahia & MNRJ 14021, 32138 & Museum & 9 & $13^{\circ} 29^{\prime} 50.64^{\prime \prime} \mathrm{S}, 40^{\circ} 33^{\prime} 11.16^{\prime \prime} \mathrm{W}$ \\
\hline Omalonyx sp. & Southeast & Maracás, Bahia & MNRJ 14021, 32138 & Museum & 9 & $13^{\circ} 29^{\prime} 50.64^{\prime \prime} \mathrm{S}, 40^{\circ} 33^{\prime} 11.16^{\prime \prime} \mathrm{W}$ \\
\hline O. unguis & Southeast & $\begin{array}{l}\text { Mocambinho, Jaíba, } \\
\text { Minas Gerais }\end{array}$ & $\begin{array}{l}\text { Oliveira \& Almeida } 2000 . \\
\text { CMIOC } 4307\end{array}$ & $\begin{array}{l}\text { Literature } \\
\text { Museum }\end{array}$ & 8 & $16^{\circ} 6^{\prime} 53.64$ "S, $43^{\circ} 58^{\prime} 28.92^{\prime \prime} \mathrm{W}$ \\
\hline O. unguis & Southeast & $\begin{array}{c}\text { Mocambinho, Jaíba, } \\
\text { Minas Gerais }\end{array}$ & CMIOC 4307 & Museum & 8 & $16^{\circ} 6^{\prime} 53.64^{\prime \prime} \mathrm{S}, 43^{\circ} 58^{\prime} 28.92^{\prime \prime} \mathrm{W}$ \\
\hline O. matheroni & Southeast & $\begin{array}{c}\text { Caratinga Biological Station, } \\
\text { Caratinga, Minas Gerais }\end{array}$ & $\begin{array}{c}\text { Arruda et al. 2006, } \\
\text { Montresor et al. } 2008\end{array}$ & Literature & 9 & $19^{\circ} 43^{\prime} 21.36^{\prime \prime} \mathrm{S}, 41^{\circ} 48^{\prime} 21.96^{\prime \prime} \mathrm{W}$ \\
\hline O. matheroni & Southeast & $\begin{array}{l}\text { Caratinga Biological Station, } \\
\text { Caratinga, Minas Gerais }\end{array}$ & $\begin{array}{c}\text { LMSM 711, 712, 714, 731-33, } \\
744-45,747-48,763 \\
\end{array}$ & Survey & 9 & $19^{\circ} 43^{\prime} 21.36^{\prime \prime} \mathrm{S}, 41^{\circ} 48^{\prime} 21.96^{\prime \prime} \mathrm{W}$ \\
\hline O. matheroni & Southeast & $\begin{array}{c}\text { Caratinga Biological Station, } \\
\text { Caratinga, Minas Gerais }\end{array}$ & MZSP 35387 & Museum & 9 & $19^{\circ} 43^{\prime} 21.36^{\prime \prime} \mathrm{S}, 41^{\circ} 48^{\prime} 21.96^{\prime \prime} \mathrm{W}$ \\
\hline Omalonyx sp. & Southeast & Teófilo Otoni, Minas Gerais & $\begin{array}{c}\text { MZSP 18442, 31886; } \\
\text { MNRJ 18365 } \\
\end{array}$ & Museum & 9 & $17^{\circ} 42^{\prime} 55.08^{\prime \prime} \mathrm{S}, 41^{\circ} 23^{\prime} 24^{\prime \prime} \mathrm{W}$ \\
\hline O. matheroni & Southeast & $\begin{array}{l}\text { Pampulha Lake, Belo } \\
\text { Horizonte, Minas Gerais }\end{array}$ & $\begin{array}{c}\text { LMSM 1378-87, 2383, 2516, } \\
\text { 2525, 2527, 2529, 2536, 2787, } \\
\text { 2918, 2929, 3153-55, 3162- } \\
\text { 63, 3166, 3186, 3208-10; } \\
\text { MCP 09277-9,09192 }\end{array}$ & Survey & 8 & $19^{\circ} 54^{\prime} 10.08^{\prime \prime S}, 43^{\circ} 57^{\prime} 36.72^{\prime \prime} \mathrm{W}$ \\
\hline O. matheroni & Southeast & $\begin{array}{c}\text { Rio Doce State Park, Marliéria, } \\
\text { Minas Gerais }\end{array}$ & $\begin{array}{c}\text { LMSM 1962-68, 2104-06, 2108; } \\
\text { MNRJ 18365 }\end{array}$ & Survey & 9 & $19^{\circ} 38^{\prime} 41.64^{\prime \prime} \mathrm{S}, 42^{\circ} 32^{\prime} 9.6^{\prime \prime} \mathrm{W}$ \\
\hline Omalonyx sp. & Southeast & $\begin{array}{c}\text { Rio Doce State Park, Marliéria, } \\
\text { Minas Gerais }\end{array}$ & MNRJ 18365 & Museum & 9 & $19^{\circ} 38^{\prime} 41.64^{\prime \prime} \mathrm{S}, 42^{\circ} 32^{\prime} 9.6^{\prime \prime} \mathrm{W}$ \\
\hline O. matheroni & Southeast & Januária, Minas Gerais & LMSM 2526-27 & Survey & 8 & $15^{\circ} 19^{\prime} 13.8^{\prime \prime} \mathrm{S}, 44^{\circ} 50^{\prime} 55.32^{\prime \prime} \mathrm{W}$ \\
\hline O. matheroni & Southeast & Nova Serrana, Minas Gerais & LMSM 713, 746 & Survey & 8 & $19^{\circ} 51^{\prime} 8.64$ ”S, $44^{\circ} 58^{\prime} 27.84^{\prime \prime} \mathrm{W}$ \\
\hline O. matheroni & Southeast & Ipatinga, Minas Gerais & LMSM 3046 & Survey & 9 & $19^{\circ} 26^{\prime} 20.76^{\prime \prime} \mathrm{S}, 42^{\circ} 36^{\prime} 14.4^{\prime \prime} \mathrm{W}$ \\
\hline O. matheroni & Southeast & Betim, Minas Gerais & $\begin{array}{c}\text { LMSM 3079-81, 3097-99, 3101, } \\
3105,3147-48,3164-65\end{array}$ & Survey & 8 & $19^{\circ} 56^{\prime} 51^{\prime \prime} \mathrm{S}, 44^{\circ} 11^{\prime} 57.84^{\prime \prime} \mathrm{W}$ \\
\hline Omalonyx sp. & Southeast & $\begin{array}{c}\text { Cachoeira Dourada, } \\
\text { Minas Gerais } \\
\end{array}$ & CMIOC 7098 & Museum & 10 & $18^{\circ} 36^{\prime} 25.92^{\prime \prime} \mathrm{S}, 49^{\circ} 28^{\prime} 48.36^{\prime \prime} \mathrm{W}$ \\
\hline
\end{tabular}


Coscarelli, D. et al.

Table 2. Continued...

\begin{tabular}{|c|c|c|c|c|c|c|}
\hline Species & Region & Locality & Record & ${ }^{1}$ Source & $\begin{array}{l}{ }^{2} \text { Hydrographic } \\
\text { Region }\end{array}$ & Coordinates \\
\hline Omalonyx sp. & Southeast & $\begin{array}{c}\text { Ilha dos Frades District, Vitoria, } \\
\text { Espírito Santo }\end{array}$ & MZSP 31965 & Museum & 9 & $20^{\circ} 18^{\prime} 7.92 ” S, 40^{\circ} 16^{\prime} 40.44 ” \mathrm{~W}$ \\
\hline Omalonyx sp. & Southeast & Serra, Espírito Santo & $\begin{array}{c}\text { LMSM } 3027 \\
\text { (Donation from CMIOC) }\end{array}$ & Donation & 9 & $20^{\circ} 7 ’ 41.16^{\prime \prime} \mathrm{S}, 40^{\circ} 18^{\prime} 6.48^{\prime \prime} \mathrm{W}$ \\
\hline Omalonyx sp. & Southeast & Rio de Janeiro, Rio de Janeiro & MNRJ 32143; 9617 & Museum & 9 & $22^{\circ} 55^{\prime} 24.24^{\prime \prime} \mathrm{S}, 43^{\circ} 26^{\prime} 51.36^{\prime \prime} \mathrm{W}$ \\
\hline O. unguis & Southeast & Rio de Janeiro, Rio de Janeiro & Lutz 1921 & Literature & 9 & $22^{\circ} 55^{\prime} 24.24^{\prime \prime} \mathrm{S}, 43^{\circ} 26^{\prime} 51.36^{\prime \prime} \mathrm{W}$ \\
\hline O. unguis & Southeast & Rio de Janeiro, Rio de Janeiro & $\begin{array}{c}\text { MNRJ 1916, 2199, 57865, } \\
12378-79 ; 12381 \\
\end{array}$ & Museum & 9 & $22^{\circ} 55^{\prime} 24.24^{\prime \prime} \mathrm{S}, 43^{\circ} 26^{\prime} 51.36^{\prime \prime} \mathrm{W}$ \\
\hline O. convexus & Southeast & Rio de Janeiro, Rio de Janeiro & $\begin{array}{l}\text { MCZ unnumber (Parodiz 1963); } \\
\text { MNHN, Gaudichaud coll. 1833 } \\
\text { (Tillier 1981) }\end{array}$ & Literature & 9 & $22^{\circ} 55^{\prime} 24.24^{\prime \prime} \mathrm{S}, 43^{\circ} 26^{\prime} 51.36^{\prime \prime} \mathrm{W}$ \\
\hline O. matheroni & Southeast & Rio de Janeiro, Rio de Janeiro & $\begin{array}{l}\text { LMSM 412-16, 3512-16 } \\
\text { (Donation from CMIOC). }\end{array}$ & Donation & 9 & $22^{\circ} 55^{\prime} 24.24^{\prime \prime} \mathrm{S}, 43^{\circ} 26^{\prime} 51.36^{\prime \prime} \mathrm{W}$ \\
\hline O. unguis & Southeast & Araruama, Rio de Janeiro & MNRJ 12380 & Museum & 9 & $22^{\circ} 45^{\prime} 16.92^{\prime \prime} \mathrm{S}, 42^{\circ} 17^{\prime} 36.24^{\prime \prime} \mathrm{W}$ \\
\hline Omalonyx sp. & Southeast & Parati, Rio de Janeiro & $\begin{array}{c}\text { LMSM } 419 \\
\text { (Donation from CMIOC) }\end{array}$ & Donation & 9 & $23^{\circ} 8^{\prime} 56.76^{\prime} \mathrm{S}, 44^{\circ} 42^{\prime} 20.16^{\prime \prime} \mathrm{W}$ \\
\hline Omalonyx sp. & Southeast & Ilha Grande, Rio de Janeiro & $\begin{array}{c}\text { LMSM 420-421 } \\
\text { (Donation from CMIOC) }\end{array}$ & Donation & 9 & 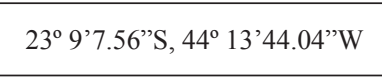 \\
\hline Omalonyx sp. & Southeast & Macaé, Rio de Janeiro & $\begin{array}{l}\text { LMSM 422-25, 431-32 (Donation } \\
\text { from CMIOC). MNRJ30737 }\end{array}$ & Donation & 9 & $22^{\circ} 17^{\prime} 42^{\prime \prime} \mathrm{S}, 41^{\circ} 58^{\prime} 29.64^{\prime \prime} \mathrm{W}$ \\
\hline Omalonyx sp. & Southeast & Raiz da Serra, Rio de Janeiro & $\begin{array}{c}\text { LMSM 417, 426-28 } \\
\text { (Donation from CMIOC) }\end{array}$ & Donation & 9 & $22^{\circ} 34^{\prime} 12^{\prime \prime} \mathrm{S}, 43^{\circ} 11^{\prime} 12.48^{\prime \prime} \mathrm{W}$ \\
\hline O. matheroni & Southeast & $\begin{array}{c}\text { Duque de Caxias, } \\
\text { Rio de Janeiro }\end{array}$ & $\begin{array}{c}\text { LMSM 841, 857-58 } \\
\text { (Donation from CMIOC) }\end{array}$ & Donation & 9 & $22^{\circ} 37^{\prime} 56.28^{\prime \prime} \mathrm{S}, 43^{\circ} 18^{\prime} 0^{\prime \prime} \mathrm{W}$ \\
\hline Omalonyx sp. & Southeast & $\begin{array}{c}\text { Pendotiba, Niterói, } \\
\text { Rio de Janeiro }\end{array}$ & CMIOC3877 & Museum & 9 & $22^{\circ} 54^{\prime} 45^{\prime \prime} \mathrm{S}, 43^{\circ} 42^{\prime} 28.8 \mathrm{~W}$ ' \\
\hline Omalonyx sp. & Southeast & São Fidélis, Rio de Janeiro & CMIOC 7515 & Museum & 9 & $21^{\circ} 39^{\prime} 36^{\prime \prime} \mathrm{S}, 41^{\circ} 47^{\prime} 16.08^{\prime \prime} \mathrm{W}$ \\
\hline Omalonyx sp. & Southeast & Pinheiral, Rio de Janeiro & MCP 09187-8 & Museum & 9 & $22^{\circ} 32^{\prime} 31.56^{\prime \prime} \mathrm{S}, 43^{\circ} 59^{\prime} 57.84^{\prime \prime} \mathrm{W}$ \\
\hline Omalonyx sp. & Southeast & $\begin{array}{c}\text { Lagoa do Campelo, Campos, } \\
\text { Rio de Janeiro }\end{array}$ & MNRJ 32139 & Museum & 9 & $21^{\circ} 39^{\prime} 10.8^{\prime \prime} \mathrm{S}, 41^{\circ} 9{ }^{\prime} 59.4^{\prime \prime} \mathrm{W}$ \\
\hline Omalonyx sp. & Southeast & Carmo, Rio de Janeiro & MNRJ1911 & Museum & 9 & $21^{\circ} 54^{\prime} 7.56^{\prime \prime} \mathrm{S}, 42^{\circ} 34^{\prime} 8.4^{\prime \prime} \mathrm{W}$ \\
\hline O. convexus & Southeast & $\begin{array}{c}\text { Campo dos Goytacazes, } \\
\text { Rio de Janeiro } \\
\end{array}$ & MNRJ 30735, 30785 & Museum & 9 & $21^{\circ} 45^{\prime} 44.28^{\prime \prime} \mathrm{S}, 41^{\circ} 19^{\prime} 44.4^{\prime \prime} \mathrm{W}$ \\
\hline Omalonyx sp. & Southeast & $\begin{array}{c}\text { Campos dos Goytacazes, } \\
\text { Rio de Janeiro } \\
\end{array}$ & MNRJ 30735, 30785 & Museum & 9 & $21^{\circ} 45^{\prime} 44.28^{\prime \prime} \mathrm{S}, 41^{\circ} 19^{\prime} 44.4^{\prime \prime} \mathrm{W}$ \\
\hline Omalonyx sp. & Southeast & $\begin{array}{c}\text { Santo Antônio de Pádua, } \\
\text { Rio de Janeiro }\end{array}$ & MNRJ 32141 & Museum & 9 & $21^{\circ} 33^{\prime} 24.84$ ”S, 42 $11^{\circ} 33.72^{\prime \prime} \mathrm{W}$ \\
\hline Omalonyx sp. & Southeast & Itaguaí, Rio de Janeiro & MNRJ 32144 & Museum & 9 & $22^{\circ} 50 ’ 29.4 ” S, 43^{\circ} 49^{\prime} 12^{\prime \prime} \mathrm{W}$ \\
\hline Omalonyx sp. & Southeast & Cambuci, Rio de Janeiro & MNRJ 32148 & Museum & 9 & $21^{\circ} 29^{\prime} 48.12^{\prime \prime S}, 41^{\circ} 54^{\prime} 52.2^{\prime \prime} \mathrm{W}$ \\
\hline Omalonyx sp. & Southeast & Osasco, São Paulo & MZSP 17071 & Museum & 10 & $23^{\circ} 31^{\prime} 44.04^{\prime \prime} \mathrm{S}, 46^{\circ} 47^{\prime} 20.76^{\prime \prime} \mathrm{W}$ \\
\hline Omalonyx sp. & Southeast & $\begin{array}{l}\text { São José do Rio Preto, } \\
\text { São Paulo }\end{array}$ & $\begin{array}{l}\text { MZSP 18441, 31878; } \\
\text { MNRJ 32137, } 14024\end{array}$ & Museum & 10 & $20^{\circ} 47^{\prime} 51^{\prime \prime} \mathrm{S}, 49^{\circ} 21^{\prime} 29.88^{\prime \prime} \mathrm{W}$ \\
\hline O. unguis & Southeast & $\begin{array}{c}\text { São José do Rio Preto, } \\
\text { São Paulo }\end{array}$ & $\begin{array}{l}\text { MZSP 18441, 31878; } \\
\text { MNRJ 32137, } 14024\end{array}$ & Museum & 10 & $20^{\circ} 47^{\prime} 51^{\prime \prime} \mathrm{S}, 49^{\circ} 21^{\prime} 29.88^{\prime \prime} \mathrm{W}$ \\
\hline Omalonyx sp. & Southeast & Caçapava, São Paulo & MZSP 31865 & Museum & 10 & $23^{\circ}$ 6'12.6”S, 45 42'48.24”'W \\
\hline Omalonyx sp. & Southeast & Barueri, São Paulo & $\begin{array}{c}\text { MZSP 31875, 47749-51, } \\
47819-50,47879\end{array}$ & Museum & 10 & $23^{\circ} 30^{\prime} 18.72^{\prime \prime} \mathrm{S}, 46^{\circ} 52^{\prime} 35.4^{\prime \prime} \mathrm{W}$ \\
\hline Omalonyx sp. & Southeast & Tremembé, São Paulo & MZSP 31876 & Museum & 9 & $22^{\circ} 56^{\prime} 25.8^{\prime \prime} \mathrm{S}, 45^{\circ} 36^{\prime} 13.32^{\prime \prime} \mathrm{W}$ \\
\hline Omalonyx sp. & Southeast & Lins, São Paulo & MZSP 31966 & Museum & 10 & $21^{\circ} 38^{\prime} 58.92^{\prime \prime} \mathrm{S}, 49^{\circ} 40^{\prime} 59.16^{\prime \prime} \mathrm{W}$ \\
\hline Omalonyx sp. & Southeast & Colômbia, São Paulo & MZSP 32664 & Museum & 10 & $20^{\circ} 15^{\prime} 59.76^{\prime \prime} \mathrm{S}, 48^{\circ} 43^{\prime} 16.32^{\prime \prime} \mathrm{W}$ \\
\hline Omalonyx sp. & Southeast & Taubaté, São Paulo & $\begin{array}{c}\text { MZSP 45129, 47718-26, 47738-47, } \\
\text { 47858-67, 47907-10, 47939-82, } \\
\text { 47995-8017, 48049, } 48053 \\
\end{array}$ & Museum & 9 & $23^{\circ} 52^{\prime} 48^{\prime \prime} \mathrm{S}, 45^{\circ} 30^{\prime} 7.92^{\prime \prime} \mathrm{W}$ \\
\hline Omalonyx sp. & Southeast & Paulínia, São Paulo & MZSP 47727-37, 47752-66 & Museum & 10 & $22^{\circ} 44^{\prime} 51.72^{\prime \prime} \mathrm{S}, 47^{\circ} 8$ '41.64"'W \\
\hline Omalonyx sp. & Southeast & Americana, São Paulo & MZSP 47938 & Museum & 10 & $22^{\circ} 43^{\prime} 23.52^{\prime \prime} \mathrm{S}, 47^{\circ} 17^{\prime} 20.04^{\prime \prime} \mathrm{W}$ \\
\hline Omalonyx sp. & Southeast & Santo André, São Paulo & MZSP 32774 (Eduardo et al. 2012) & Literature & 10 & $23^{\circ} 43^{\prime} 41.88^{\prime \prime} \mathrm{S}, 46^{\circ} 26^{\prime} 28.68^{\prime \prime} \mathrm{W}$ \\
\hline O. matheroni & Southeast & Santo André, São Paulo & MZSP 32774 (Eduardo et al. 2012) & Literature & 10 & $23^{\circ} 43^{\prime} 41.88^{\prime \prime} \mathrm{S}, 46^{\circ} 26^{\prime} 28.68^{\prime \prime} \mathrm{W}$ \\
\hline O. matheroni & Southeast & $\begin{array}{l}\text { Ibitinga Powerplant, Ibitinga, } \\
\text { São Paulo }\end{array}$ & MCP 09120 (Arruda et al. 2009) & Literature & 10 & $21^{\circ} 45^{\prime} 28.08^{\prime \prime S}, 48^{\circ} 59 ’ 30.12^{\prime \prime} \mathrm{W}$ \\
\hline O. matheroni & $\begin{array}{c}\text { Central } \\
\text { West }\end{array}$ & $\begin{array}{c}\text { Santa Rita do Novo Destino, } \\
\text { Goiás }\end{array}$ & LMSM 3241-42 & Donation & 8 & $14^{\circ} 52^{\prime} 35.4^{\prime \prime} \mathrm{S}, 49^{\circ} 33^{\prime} 46.8^{\prime \prime} \mathrm{W}$ \\
\hline
\end{tabular}


Table 2. Continued...

\begin{tabular}{|c|c|c|c|c|c|c|}
\hline Species & Region & Locality & Record & ${ }^{1}$ Source & $\begin{array}{c}{ }^{2} \text { Hydrographic } \\
\text { Region }\end{array}$ & Coordinates \\
\hline Omalonyx sp. & $\begin{array}{c}\text { Central } \\
\text { West }\end{array}$ & $\begin{array}{l}\text { Passo da Lontra, } \\
\text { Mato Grosso do Sul }\end{array}$ & MZSP 41206 & Museum & 10 & $19^{\circ} 34^{\prime} 29.28^{\prime \prime S}, 57^{\circ} 22^{\prime} 40.8^{\prime \prime} \mathrm{W}$ \\
\hline Omalonyx sp. & $\begin{array}{c}\text { Central } \\
\text { West }\end{array}$ & $\begin{array}{c}\text { Três Lagoas, } \\
\text { Mato Grosso do Sul } \\
\end{array}$ & MZSP 47748 & Museum & 10 & $20^{\circ} 26^{\prime} 19.68^{\prime \prime S}, 52^{\circ} 12^{\prime} 41.04^{\prime \prime} \mathrm{W}$ \\
\hline O. unguis & $\begin{array}{c}\text { Central } \\
\text { West }\end{array}$ & $\begin{array}{c}\text { Campo Grande, } \\
\text { Mato Grosso do Sul }\end{array}$ & LMSM 2747 & Survey & 10 & $20^{\circ} 54^{\prime} 47.16^{\prime \prime S}, 54^{\circ} 14^{\prime} 58.92^{\prime \prime} \mathrm{W}$ \\
\hline O. unguis & $\begin{array}{l}\text { Central } \\
\text { West }\end{array}$ & Miranda, Mato Grosso do Sul & $\begin{array}{c}\text { LMSM } 2705-07,2739-42,2769, \\
2780-81,2788,2797-99,2898-99, \\
2900-08,2911-13,2917\end{array}$ & Survey & 10 & $20^{\circ} 7^{\prime} 12.36^{\prime \prime} \mathrm{S}, 56^{\circ} 35^{\prime} 52.8^{\prime \prime} \mathrm{W}$ \\
\hline O. unguis & $\begin{array}{c}\text { Central } \\
\text { West }\end{array}$ & $\begin{array}{l}\text { Transpantaneira Road, } \\
\text { Mato Grosso }\end{array}$ & MNRJ 9969 & Museum & 10 & $16^{\circ} 22^{\prime} 30^{\prime} \mathrm{S}, 56^{\circ} 40^{\prime} 12^{\prime \prime} \mathrm{W}$ \\
\hline O. unguis & $\begin{array}{c}\text { Central } \\
\text { West }\end{array}$ & $\begin{array}{c}\text { Transpantaneira Road, Poconé, } \\
\text { Mato Grosso }\end{array}$ & $\begin{array}{l}\text { LMSM 2673; LMSM 429-430 } \\
\text { (Donation from CMIOC). }\end{array}$ & Donation & 10 & $16^{\circ} 22^{\prime} 30^{\prime \prime} \mathrm{S}, 56^{\circ} 40^{\prime} 12^{\prime \prime} \mathrm{W}$ \\
\hline O. unguis & $\begin{array}{c}\text { Central } \\
\text { West }\end{array}$ & Cáceres, Mato Grosso & LMSM 3334 & Survey & 10 & $16^{\circ} 31^{\prime} 26.4^{\prime \prime} \mathrm{S}, 57^{\circ} 50^{\prime} 16.8^{\prime \prime} \mathrm{W}$ \\
\hline O. unguis & $\begin{array}{c}\text { Central } \\
\text { West }\end{array}$ & Várzea Grande, Mato Grosso & LMSM 3344 & Survey & 10 & $15^{\circ} 33^{\prime} 23.4^{\prime \prime} \mathrm{S}, 56^{\circ} 16^{\prime} 53.04^{\prime \prime} \mathrm{W}$ \\
\hline \multirow[t]{2}{*}{ Omalonyx sp. } & \multirow{2}{*}{$\begin{array}{c}\text { Central } \\
\text { West }\end{array}$} & \multirow[t]{2}{*}{ Barão de Melgaço, Mato Grosso } & $\begin{array}{c}\text { LMSM } 433 \\
\text { (Donation from CMIOC). }\end{array}$ & Donation & \multirow[t]{2}{*}{10} & \multirow[t]{2}{*}{$16^{\circ} 49^{\prime} 14.52^{\prime \prime} \mathrm{S}, 56^{\circ} 19^{\prime} 19.2^{\prime \prime} \mathrm{W}$} \\
\hline & & & CMIOC 9565 & Museum & & \\
\hline Omalonyx sp. & $\begin{array}{c}\text { Central } \\
\text { West }\end{array}$ & Barão de Melgaço, Mato Grosso & CMIOC 9565 & Museum & 10 & $16^{\circ} 49^{\prime} 14.52^{\prime \prime S}, 56^{\circ} 19^{\prime} 19.2^{\prime \prime} \mathrm{W}$ \\
\hline Omalonyx sp. & $\begin{array}{c}\text { Central } \\
\text { West }\end{array}$ & Manso Lake, Mato Grosso & $\begin{array}{c}\text { LMSM 784-86, } 791 \\
\text { (Donation from CMIOC) }\end{array}$ & Donation & 10 & $15^{\circ} 26^{\prime} 20.4^{\prime \prime} \mathrm{S}, 55^{\circ} 11^{\prime} 9.96^{\prime \prime} \mathrm{W}$ \\
\hline Omalonyx sp. & $\begin{array}{c}\text { Central } \\
\text { West }\end{array}$ & $\begin{array}{c}\text { Chapada dos Guimarães, } \\
\text { Mato Grosso } \\
\end{array}$ & $\begin{array}{c}\text { LMSM } 3032 \\
\text { (Donation from CMIOC) } \\
\end{array}$ & Donation & 10 & $15^{\circ} 6^{\prime} 30.24^{\prime \prime} \mathrm{S}, 55^{\circ} 32^{\prime} 22.92^{\prime \prime} \mathrm{W}$ \\
\hline Omalonyx sp. & $\begin{array}{c}\text { Central } \\
\text { West }\end{array}$ & Nobres, Mato Grosso & $\begin{array}{c}\text { LMSM 3028, } 3030 \\
\text { (Donation from CMIOC) }\end{array}$ & Donation & 10 & $14^{\circ} 21^{\prime} 51.12^{\prime \prime} \mathrm{S}, 55^{\circ} 46^{\prime} 48^{\prime \prime} \mathrm{W}$ \\
\hline Omalonyx sp. & $\begin{array}{c}\text { Central } \\
\text { West }\end{array}$ & $\begin{array}{l}\text { Santo Antônio do Leverger, } \\
\text { Mato Grosso }\end{array}$ & $\begin{array}{c}\text { LMSM } 3029 \\
\text { (Donation from CMIOC) }\end{array}$ & Donation & 10 & $16^{\circ} 27^{\prime} 23.4^{\prime \prime} \mathrm{S}, 55^{\circ} 26^{\prime} 22.92^{\prime \prime} \mathrm{W}$ \\
\hline O. matheroni & $\begin{array}{c}\text { Central } \\
\text { West }\end{array}$ & Barra do Garças, Mato Grosso & МСР 09189 & Museum & 10 & $15^{\circ} 21^{\prime} 34.56^{\prime \prime S}, 52^{\circ} 29^{\prime} 50.28^{\prime \prime} \mathrm{W}$ \\
\hline O.matheroni & South & Paranaguá, Paraná & MZSP 18440 (Arruda et al. 2009) & Literature & 12 & $25^{\circ} 32^{\prime} 39.48^{\prime \prime} \mathrm{S}, 48^{\circ} 32^{\prime} 47.4^{\prime \prime} \mathrm{W}$ \\
\hline Omalonyx sp. & South & Paranaguá, Paraná & MZSP 18440 (Arruda et al. 2009) & Literature & 12 & $25^{\circ} 32^{\prime} 39.48^{\prime \prime} \mathrm{S}, 48^{\circ} 32^{\prime} 47.4^{\prime \prime} \mathrm{W}$ \\
\hline Omalonyx sp. & South & Sete Quedas, Guaíra, Paraná & MZSP 31884 & Museum & 12 & $24^{\circ} 48^{\prime} 25.2^{\prime \prime} \mathrm{S}, 54^{\circ} 15^{\prime} 12.24^{\prime \prime} \mathrm{W}$ \\
\hline O. convexus & South & Curitiba, Paraná & $\begin{array}{c}\text { LMSM 2667, 2975, 2990-91, } \\
\quad 3003,3008,3011 \\
\end{array}$ & Survey & 12 & $25^{\circ} 28^{\prime} 41.16^{\prime \prime} \mathrm{S}, 49^{\circ} 17^{\prime} 17.16^{\prime \prime} \mathrm{W}$ \\
\hline O. unguis & South & Foz do Iguaçu, Paraná & LMSM 3260 & Survey & 12 & $25^{\circ} 28^{\prime} 15.24^{\prime \prime} \mathrm{S}, 54^{\circ} 28^{\prime} 56.28^{\prime \prime} \mathrm{W}$ \\
\hline O. unguis & South & Foz do Iguaçu, Paraná & MNRJ 32146, 32205 & Museum & 12 & $25^{\circ} 28^{\prime} 15.24^{\prime \prime} \mathrm{S}, 54^{\circ} 28^{\prime} 56.28^{\prime \prime} \mathrm{W}$ \\
\hline O. unguis & South & Foz do Iguaçu, Paraná & MNRJ 32146, 32205 & Museum & 12 & $25^{\circ} 28^{\prime} 15.24^{\prime \prime} \mathrm{S}, 54^{\circ} 28^{\prime} 56.28^{\prime \prime} \mathrm{W}$ \\
\hline O. convexus & South & Araranguá, Santa Catarina & $\begin{array}{l}\text { LMSM 1522, 2988-89, 3001-02, } \\
\text { 3005-07 }\end{array}$ & Survey & 12 & $28^{\circ} 56^{\prime} 33.36^{\prime \prime} \mathrm{S}, 49^{\circ} 28^{\prime} 22.08^{\prime \prime} \mathrm{W}$ \\
\hline O. matheroni & South & $\begin{array}{c}\text { Near to Tironi Park, Piçarras, } \\
\text { Santa Catarina }\end{array}$ & LMSM 3942, 4024-4028 & Survey & 12 & $26^{\circ} 45^{\prime} 56.88^{\prime \prime} \mathrm{S}, 48^{\circ} 42^{\prime} 38.88^{\prime \prime} \mathrm{W}$ \\
\hline O. convexus & South & São João do Sul, Santa Catarina & MCP 09271 (Agudo-Padron 2008) & Literature & 12 & $29^{\circ} 12^{\prime} 22.32^{\prime \prime} \mathrm{S}, 49^{\circ} 48^{\prime} 46.08^{\prime \prime} \mathrm{W}$ \\
\hline O. convexus & South & Camboriú, Santa Catarina & Agudo-Padron 2008 & Literature & 12 & $27^{\circ} 42^{\prime} 43.2^{\prime \prime} \mathrm{S}, 48^{\circ} 42^{\prime} 32.4^{\prime \prime} \mathrm{W}$ \\
\hline O. convexus & South & Paulo Lopez, Santa Catarina & Agudo-Padron 2008 & Literature & 12 & $27^{\circ} 57^{\prime} 53.28^{\prime \prime} \mathrm{S}, 48^{\circ} 45^{\prime} 39.6^{\prime \prime} \mathrm{W}$ \\
\hline O. convexus & South & Criciúma, Santa Catarina & Agudo-Padron 2008 & Literature & 12 & $28^{\circ} 42^{\prime} 59.04^{\prime \prime} \mathrm{S}, 49^{\circ} 22^{\prime} 49.8^{\prime \prime} \mathrm{W}$ \\
\hline O. matheroni & South & $\begin{array}{c}\text { Biguaçu River, Biguaçu, } \\
\text { Santa Catarina }\end{array}$ & LMSM 3507, 3508 & Survey & 12 & $27^{\circ} 28^{\prime} 27.84^{\prime \prime} \mathrm{S}, 48^{\circ} 40^{\prime} 9.84^{\prime \prime} \mathrm{W}$ \\
\hline O. convexus & South & Florianópolis, Santa Catarina & МСР 09190-1 & Museum & 12 & $27^{\circ} 24^{\prime} 24.12^{\prime \prime S}, 48^{\circ} 25^{\prime} 45.84^{\prime \prime} \mathrm{W}$ \\
\hline O. convexus & South & Palhoça, Santa Catarina & MCP 09227 & Museum & 12 & $27^{\circ} 46^{\prime} 44.76^{\prime} \mathrm{S}, 48^{\circ} 40^{\prime} 15.6^{\prime \prime} \mathrm{W}$ \\
\hline O. convexus & South & Porto Alegre, Rio Grande do Sul & $\begin{array}{l}\text { Martens 1868, Heynemann 1868; } \\
\text { Lange de Morretes 1949; Tillier } \\
\text { 1981, Simone 2006; MCNZ: } \\
\text { 8058, 1524, 31590, 4439, 5501, } \\
\text { 35546 (Arruda \& Thomé 2011) }\end{array}$ & Literature & 12 & $31^{\circ} 14^{\prime} 58.56^{\prime \prime} \mathrm{S}, 51^{\circ} 30^{\prime} 37.08^{\prime \prime} \mathrm{W}$ \\
\hline O. convexus & South & Porto Alegre, Rio Grande do Sul & $\begin{array}{c}\text { MCP 02066, 08834, 08837-8, } \\
08849,08842-3,08845,09273-4\end{array}$ & Museum & 12 & $31^{\circ} 14^{\prime} 58.56^{\prime \prime} \mathrm{S}, 51^{\circ} 30^{\prime} 37.08^{\prime \prime} \mathrm{W}$ \\
\hline O. convexus & South & Camaquã, Rio Grande do Sul & $\begin{array}{l}\text { Parodiz 1963; MCP } 8841 \\
\text { (Arruda \& Thomé 2011) }\end{array}$ & Literature & 12 & $30^{\circ} 55^{\prime} 21.36^{\prime \prime} \mathrm{S}, 51^{\circ} 47^{\prime} 18.6^{\prime \prime} \mathrm{W}$ \\
\hline
\end{tabular}


Coscarelli, D. et al.

Table 2. Continued..

\begin{tabular}{|c|c|c|c|c|c|c|}
\hline Species & Region & Locality & Record & ${ }^{1}$ Source & $\begin{array}{c}{ }^{2} \text { Hydrographic } \\
\text { Region }\end{array}$ & Coordinates \\
\hline Omalonyx sp. & South & Camaquã, Rio Grande do Sul & MCP 09272; MZSP 7539 & Museum & 12 & $30^{\circ} 55^{\prime} 21.36^{\prime \prime} \mathrm{S}, 51^{\circ} 47^{\prime} 18.6^{\prime \prime} \mathrm{W}$ \\
\hline O. unguis & South & $\begin{array}{c}\text { São Leopoldo, } \\
\text { Rio Grande do Sul }\end{array}$ & $\begin{array}{l}\text { MCP 10247, 10240-1, 10291; } \\
\text { MZSP 31961,18439 }\end{array}$ & Museum & 12 & $29^{\circ} 45^{\prime} 19.08^{\prime} \mathrm{S}, 51^{\circ} 8^{\prime} 42.72^{\prime \prime} \mathrm{W}$ \\
\hline Omalonyx sp. & South & $\begin{array}{c}\text { São Leopoldo, } \\
\text { Rio Grande do Sul }\end{array}$ & $\begin{array}{l}\text { MCP 10247, 10240-1, 10291; } \\
\text { MZSP 31961,18439 }\end{array}$ & Museum & 12 & $29^{\circ} 45^{\prime} 19.08^{\prime \prime} \mathrm{S}, 51^{\circ} 8^{\prime} 42.72^{\prime \prime} \mathrm{W}$ \\
\hline Omalonyx sp. & South & Pelotas, Rio Grande do Sul & MZSP 31964 & Museum & 12 & $31^{\circ} 34^{\prime} 7.68^{\prime \prime} \mathrm{S}, 52^{\circ} 21^{\prime} 38.16^{\prime \prime} \mathrm{W}$ \\
\hline O. convexus & South & Rio Grande, Rio Grande do Sul & $\begin{array}{c}\text { MCP } 08836 \\
\text { (Arruda \& Thomé 2011) }\end{array}$ & Literature & 12 & $32^{\circ} 13^{\prime} 10.92^{\prime \prime S}, 52^{\circ} 24^{\prime} 15.48^{\prime \prime} \mathrm{W}$ \\
\hline O. convexus & South & Viamão, Rio Grande do Sul & $\begin{array}{c}\text { LMSM 2650-51, 2666, 2668-69, } \\
2704,2759,2762-63,2768 \\
2895-97\end{array}$ & Survey & 12 & $30^{\circ} 10^{\prime} 12^{\prime} \mathrm{S}, 50^{\circ} 52^{\prime} 10.56^{\prime \prime} \mathrm{W}$ \\
\hline O. convexus & South & Viamão, Rio Grande do Sul & $\begin{array}{c}\text { MCNZ 2506 } \\
\text { (Arruda \& Thomé, 2011) }\end{array}$ & Literature & 12 & $30^{\circ} 10^{\prime} 12^{\prime \prime} \mathrm{S}, 50^{\circ} 52^{\prime} 10.56^{\prime \prime} \mathrm{W}$ \\
\hline O. convexus & South & Arambaré, Rio Grande do Sul & $\begin{array}{l}\text { LMSM 3009-10, 3021-23, 3149- } \\
\quad 50,3167,3175,3187 \\
\end{array}$ & Survey & 12 & $30^{\circ} 55^{\prime} 9.12^{\prime \prime} \mathrm{S}, 51^{\circ} 34^{\prime} 42.6^{\prime \prime} \mathrm{W}$ \\
\hline O. convexus & South & $\begin{array}{l}\text { Cachoeira do Sul, } \\
\text { Rio Grande do Sul }\end{array}$ & $\begin{array}{c}\text { MCP 08840 } \\
\text { (Arruda \& Thomé 2011) }\end{array}$ & Literature & 12 & $30^{\circ} 12^{\prime} 22.32^{\prime \prime} \mathrm{S}, 52^{\circ} 59^{\prime} 11.04$ ”W \\
\hline O. convexus & South & Santa Maria, Rio Grande do Sul & $\begin{array}{c}\text { MCP 08829-31 } \\
\text { (Arruda \& Thomé 2011) }\end{array}$ & Literature & 12 & $29^{\circ} 47^{\prime} 56.4^{\prime \prime S}, 53^{\circ} 49^{\prime} 29.28^{\prime \prime} \mathrm{W}$ \\
\hline O. convexus & South & $\begin{array}{l}\text { Novo Hamburgo, } \\
\text { Rio Grande do Sul }\end{array}$ & $\begin{array}{c}\text { MCP } 08848 \\
\text { (Arruda \& Thomé 2011) }\end{array}$ & Literature & 12 & $29^{\circ} 44^{\prime} 6.72^{\prime} \mathrm{S}, 51^{\circ} 29^{\prime} 13.2^{\prime \prime} \mathrm{W}$ \\
\hline O. convexus & South & $\begin{array}{c}\text { Mampituba Stream, Torres, } \\
\text { Rio Grande do Sul }\end{array}$ & $\begin{array}{c}\text { MCP } 08832 \\
\text { (Arruda \& Thomé 2011); }\end{array}$ & Literature & 12 & $29^{\circ} 35^{\prime} 29.76^{\prime} \mathrm{S}, 49^{\circ} 57^{\prime} 36^{\prime \prime} \mathrm{W}$ \\
\hline O. convexus & South & $\begin{array}{c}\text { Mampituba Stream, Torres, } \\
\text { Rio Grande do Sul }\end{array}$ & LMSM 3932-3941 & Survey & & $29^{\circ} 35^{\prime} 29.76^{\prime} \mathrm{S}, 49^{\circ} 57^{\prime} 36^{\prime \prime} \mathrm{W}$ \\
\hline O. convexus & South & $\begin{array}{l}\text { Cachoeirinha, } \\
\text { Rio Grande do Sul }\end{array}$ & $\begin{array}{c}\text { MCP 09548 (Agudo } \\
\text { Padron 2012); MCP 08839 } \\
\text { (Arruda \& Thomé 2011) }\end{array}$ & Literature & 12 & $29^{\circ} 55^{\prime} 15.96 ” S, 51^{\circ} 56^{\prime} 56.4^{\prime \prime} \mathrm{W}$ \\
\hline O. convexus & South & Vacaria, Rio Grande do Sul & $\begin{array}{c}\text { MCNZ } 7559 \\
\text { (Arruda \& Thomé 2011) }\end{array}$ & Literature & 12 & $28^{\circ} 22^{\prime} 14.88^{\prime \prime} \mathrm{S}, 50^{\circ} 55^{\prime} 24.96$ ”W \\
\hline O. convexus & South & Sapiranga, Rio Grande do Sul & $\begin{array}{c}\text { MCNZ } 2877 \\
\text { (Arruda \& Thomé 2011) }\end{array}$ & Literature & 12 & $29^{\circ} 36^{\prime} 50.4^{\prime \prime} \mathrm{S}, 50^{\circ} 59^{\prime} 43.8^{\prime \prime} \mathrm{W}$ \\
\hline O. convexus & South & Guaíba, Rio Grande do Sul & $\begin{array}{c}\text { MCNZ } 6000 \\
\text { (Arruda \& Thomé 2011) }\end{array}$ & Literature & 12 & $30^{\circ} 10^{\prime} 39.36^{\prime \prime} \mathrm{S}, 51^{\circ} 26^{\prime} 9.6^{\prime \prime} \mathrm{W}$ \\
\hline \multirow[t]{2}{*}{ O. convexus } & \multirow[t]{2}{*}{ South } & \multirow[t]{2}{*}{ Triunfo, Rio Grande do Sul } & $\begin{array}{c}\text { MCNZ } 8058 \\
\text { (Arruda \& Thomé 2011) }\end{array}$ & Literature & \multirow[t]{2}{*}{12} & \multirow[t]{2}{*}{$29^{\circ} 50^{\prime} 37.32^{\prime \prime S}, 51^{\circ} 34^{\prime} 17.04^{\prime \prime} \mathrm{W}$} \\
\hline & & & MCP 06487 & Museum & & \\
\hline O. convexus & South & Triunfo, Rio Grande do Sul & MCP 06487 & Museum & 12 & $29^{\circ} 50^{\prime} 37.32^{\prime \prime S}, 51^{\circ} 34^{\prime} 17.04^{\prime \prime} \mathrm{W}$ \\
\hline O. unguis & South & Imbé, Rio Grande do Sul & $\begin{array}{c}\text { MCNZ } 31969 \\
\text { (Arruda \& Thomé 2011) }\end{array}$ & Literature & 12 & $29^{\circ} 55^{\prime} 50.16^{\prime \prime} \mathrm{S}, 50^{\circ} 77^{\prime} 55.2^{\prime \prime} \mathrm{W}$ \\
\hline O. convexus & South & $\begin{array}{c}\text { Capão do Corvo, Canoas, } \\
\text { Rio Grande do Sul } \\
\end{array}$ & $\begin{array}{c}\text { MCNZ } 597 \\
\text { (Arruda \& Thomé 2011) }\end{array}$ & Literature & 12 & $29^{\circ} 54^{\prime} 48.24^{\prime \prime} \mathrm{S}, 51^{\circ} 10^{\prime} 37.2^{\prime \prime} \mathrm{W}$ \\
\hline O. convexus & South & Taquara, Rio Grande do Sul & $\begin{array}{c}\text { MCNZ } 2610 \\
\text { (Arruda \& Thomé 2011) }\end{array}$ & Literature & 12 & $29^{\circ} 40^{\prime} 15.6^{\prime \prime} \mathrm{S}, 50^{\circ} 45^{\prime} 47.52^{\prime \prime} \mathrm{W}$ \\
\hline O. convexus & South & Portão, Rio Grande do Sul & $\begin{array}{c}\text { MCNZ } 3264 \\
\text { (Arruda \& Thomé 2011) }\end{array}$ & Literature & 12 & $29^{\circ} 42^{\prime} 36^{\prime} \mathrm{S}, 51^{\circ} 14^{\prime} 56.76^{\prime \prime} \mathrm{W}$ \\
\hline O. convexus & South & Estrela, Rio Grande do Sul & $\begin{array}{c}\text { MCP 08844 } \\
\text { (Arruda \& Thomé 2011) }\end{array}$ & Literature & 12 & $29^{\circ} 30^{\prime} 33.48^{\prime \prime} \mathrm{S}, 51^{\circ} 55^{\prime} 11.64^{\prime \prime} \mathrm{W}$ \\
\hline O. convexus & South & $\begin{array}{c}\text { Eldorado do Sul, } \\
\text { Rio Grande do Sul }\end{array}$ & MCP 08835 & Museum & 12 & $30^{\circ} 46^{\prime} 58.8^{\prime \prime} \mathrm{S}, 51^{\circ} 29^{\prime} 54.24^{\prime \prime} \mathrm{W}$ \\
\hline Omalonyx sp. & South & São Borja, Rio Grande do Sul & $\begin{array}{c}\text { MCP 09270; MCP 08828; } \\
\text { МCP } 08835\end{array}$ & Museum & 10 & $28^{\circ} 44^{\prime} 46.68^{\prime \prime} \mathrm{S}, 55^{\circ} 47^{\prime} 50.28^{\prime \prime} \mathrm{W}$ \\
\hline O. convexus & South & Mampituba, Rio Grande do Sul & MCP 08833 & Museum & 12 & $29^{\circ} 15^{\prime} 43.92^{\prime \prime} \mathrm{S}, 50^{\circ} 13^{\prime} 12^{\prime \prime} \mathrm{W}$ \\
\hline O. brasiliensis & South & Rio Grande do Sul & $\begin{array}{c}\text { ZMB } 45.913 \\
\text { (Arruda \& Thomé 2008a). }\end{array}$ & Literature & $10 /$ dez & $\begin{array}{c}29^{\circ} 36^{\prime} 18.24^{\prime \prime} \mathrm{S} 53^{\circ} 12^{\prime} 10.25^{\prime \prime} \mathrm{W} \\
\text { unespecified locality }\end{array}$ \\
\hline O. brasiliensis & South & Rio Grande do Sul & MNRJ 57927, 57955 & Museum & $10 / \mathrm{dez}$ & $\begin{array}{c}29^{\circ} 36^{\prime} 18.24^{\prime \prime S ~} 53^{\circ} 12^{\prime} 10.25^{\prime \prime} \mathrm{W} \\
\text { unespecified locality }\end{array}$ \\
\hline
\end{tabular}


Table 3. Records of Omalonyx in South America and Lesser Antilles based on literature data, institutional collections and our own malacological surveys.

\begin{tabular}{|c|c|c|c|c|c|c|}
\hline Species & Country & Locality & ${ }^{1}$ Record & ${ }^{2}$ Source & $\begin{array}{c}{ }^{3} \text { Hydrographic } \\
\text { Region }\end{array}$ & Coordinates \\
\hline O. unguis & Argentina & $\begin{array}{c}\text { Rio Santiago, Buenos } \\
\text { Aires }\end{array}$ & $\begin{array}{c}\text { MACN 10218-1 (Hylton-Scott } \\
\text { 1968); MACN 10208; 10268; MLP } \\
\text { 31321 (Arruda \& Thomé 2008b), } \\
\text { Coscarelli \& Vidigal } 2011\end{array}$ & Literature & 10 & $34^{\circ} 55^{\prime} 15.96^{\prime \prime S}, 57^{\circ} 57^{\prime} 15.84^{\prime \prime} \mathrm{W}$ \\
\hline Omalonyx sp. & Argentina & $\begin{array}{c}\text { Punta Lara, Buenos } \\
\text { Aires }\end{array}$ & MZSP 31866 & Museum & 10 & $34^{\circ} 49^{\prime} 40.8^{\prime \prime} \mathrm{S}, 57^{\circ} 57^{\prime} 56.52^{\prime \prime} \mathrm{W}$ \\
\hline O. convexus & Argentina & Ezeiza, Buenos Aires & $\begin{array}{l}\text { Camaggni coll. } 1971 \text { (Tillier } \\
\text { 1981); MNHN unnumbered } \\
\text { (Arruda \& Thomé 2008b) }\end{array}$ & Literature & 10 & $34^{\circ} 51^{\prime} 13.68^{\prime \prime} \mathrm{S}, 58^{\circ} 31^{\prime} 22.44^{\prime \prime} \mathrm{W}$ \\
\hline O. convexus & Argentina & La Plata, Buenos Aires & LMSM 3273, 3237 & Survey & 10 & $34^{\circ} 55^{\prime} 15.96^{\prime \prime S}, 57^{\circ} 57^{\prime} 15.84^{\prime \prime} \mathrm{W}$ \\
\hline o. convexus & Argentina & $\begin{array}{l}\text { Rio Santiago, Buenos } \\
\text { Aires }\end{array}$ & $\begin{array}{l}\text { MNHN, unnumbered; MACN } 14472 \\
\text { (Arruda \& Thomé 2008b) }\end{array}$ & Literature & 10 & $34^{\circ} 55^{\prime} 15.96^{\prime \prime S}, 57^{\circ} 57^{\prime} 15.84^{\prime \prime} \mathrm{W}$ \\
\hline o. convexus & Argentina & Tucumán, Tucumán & $\begin{array}{c}\text { Hylton-Scott 1968, 1971; SMNH } 2507 \\
\text { (Tillier 1981); MNHN unnumbered } \\
\text { (Arruda \& Thomé 2008b) }\end{array}$ & Literature & 11 & $26^{\circ} 48^{\prime} 30.24^{\prime \prime} \mathrm{S}, 65^{\circ} 13^{\prime} 33.6^{\prime \prime} \mathrm{W}$ \\
\hline O. unguis & Argentina & Tucumán, Tucumán & MNRJ 4470 & Museum & 11 & $26^{\circ} 48^{\prime} 30.24^{\prime \prime} \mathrm{S}, 65^{\circ} 13^{\prime} 33.6^{\prime \prime} \mathrm{W}$ \\
\hline o. convexus & Argentina & Rosario, Santa Fe & $\begin{array}{c}\text { CM and MZUM unnumbered (Parodiz, } \\
\text { 1963); MACN } 26577 \text { (Hylton- } \\
\text { Scott \& Lapuente 1968); USNM } \\
\text { 124546 (Tillier 1981); ZMB28513 } \\
\text { (Arruda \& Thomé 2008b) }\end{array}$ & Literature & 10 & $32^{\circ} 56^{\prime} 40.2^{\prime \prime} \mathrm{S}, 60^{\circ} 39^{\prime} 10.8^{\prime \prime} \mathrm{W}$ \\
\hline O. convexus & Argentina & Rosario, Santa Fe & ANSP 64116 & Museum & 10 & $32^{\circ} 56^{\prime} 40.2^{\prime \prime} \mathrm{S}, 60^{\circ} 39^{\prime} 10.8^{\prime \prime} \mathrm{W}$ \\
\hline o. convexus & Argentina & Rio Negro, Chaco & $\begin{array}{l}\text { CM unnumbered (Parodiz 1963), } \\
\text { (Arruda \& Thomé 2008b) }\end{array}$ & Literature & 10 & $27^{\circ} 29^{\prime} 39.12^{\prime \prime} \mathrm{S}, 58^{\circ} 44^{\prime} 38.4^{\prime \prime} \mathrm{W}$ \\
\hline o. convexus & Argentina & $\begin{array}{c}\text { Islands in Paraná } \\
\text { River, near Santa Fe }\end{array}$ & $\begin{array}{l}\text { CM unnumbered (Parodiz 1963), } \\
\text { (Arruda \& Thomé 2008b) }\end{array}$ & Literature & 10 & $31^{\circ} 56^{\prime} 57.84^{\prime \prime S}, 60^{\circ} 27^{\prime} 28.44^{\prime \prime} \mathrm{W}$ \\
\hline Omalonyx sp. & Argentina & $\begin{array}{c}\text { Lagunas Pampeanas, } \\
\text { Buenos Aires }\end{array}$ & Tietze \& Francesco 2012 & Literature & 10 & - \\
\hline O. unguis & Argentina & $\begin{array}{l}\text { Locality between } \\
\text { Santa Fe and Paraná, } \\
\text { both in Argentina }\end{array}$ & Zilli et al. 2008 & Literature & 10 & - \\
\hline O. unguis & Argentina & $\begin{array}{l}\text { Site within Chaco } \\
\text { Province, on the west } \\
\text { bank of the Paraná } \\
\text { River at its confluence } \\
\text { with Paraguay River } \\
\end{array}$ & Franceschini et al. 2010 & Literature & 10 & - \\
\hline o. convexus & Argentina & $\begin{array}{l}\text { Abra Vieja, Paraná } \\
\text { River, Buenos Aires }\end{array}$ & $\begin{array}{c}\text { CM and MACN } \\
\text { unnumbered (Parodiz 1963), } \\
\text { (Arruda \& Thomé 2008b) }\end{array}$ & Literature & 10 & $34^{\circ} 34^{\prime} 43.32^{\prime \prime} \mathrm{S}, 58^{\circ} 48^{\prime} 15.12^{\prime \prime} \mathrm{W}$ \\
\hline O. convexus & Argentina & $\begin{array}{c}\text { Perucho Verna Stream, } \\
\text { Entre Rios }\end{array}$ & UF 159845 (Arruda \& Thomé 2008b) & Literature & 10 & $23^{\circ} 34^{\prime} 25.32^{\prime \prime} \mathrm{S}, 64^{\circ} 8^{\prime} 31.2^{\prime \prime} \mathrm{W}$ \\
\hline o. convexus & Argentina & Vinalito, Jujuy & $\begin{array}{l}\text { CM unnumbered (Parodiz 1963), } \\
\text { (Arruda \& Thomé 2008b) }\end{array}$ & Literature & 10 & $23^{\circ} 34^{\prime} 25.32^{\prime} \mathrm{S}, 64^{\circ} 8{ }^{\prime} 31.2^{\prime \prime} \mathrm{W}$ \\
\hline O. convexus & Argentina & $\begin{array}{l}\text { Santa Bárbara Ridge, } \\
\text { Jujuy }\end{array}$ & $\begin{array}{l}\text { CM unnumbered (Parodiz 1963), } \\
\text { (Arruda \& Thomé 2008b) }\end{array}$ & Literature & 10 & $23^{\circ} 46^{\prime} 33.6^{\prime \prime} \mathrm{S}, 64^{\circ} 40^{\prime} 10.56^{\prime \prime} \mathrm{W}$ \\
\hline o. convexus & Argentina & $\begin{array}{l}\text { Termas del Palmar, } \\
\text { Salta }\end{array}$ & $\begin{array}{c}\text { CM unnumbered (Parodiz } \\
\text { 1963); MACN 27246 } \\
\text { (Arruda \& Thomé 2008b) } \\
\end{array}$ & Literature & 10 & $24^{\circ} 14^{\prime} 54.96^{\prime \prime} \mathrm{S}, 63^{\circ} 27^{\prime} 12.96^{\prime \prime} \mathrm{W}$ \\
\hline o. convexus & Argentina & Orán, Salta & $\begin{array}{l}\text { CM unnumbered (Parodiz 1963), } \\
\text { (Arruda \& Thomé 2008b) }\end{array}$ & Literature & 10 & $23^{\circ} 8^{\prime} 9.96^{\prime \prime} \mathrm{S}, 64^{\circ} 19^{\prime} 19.92^{\prime \prime} \mathrm{W}$ \\
\hline O. unguis & Argentina & Resistencia, Chaco & $\begin{array}{c}\text { MACN22931 } \\
\text { (Arruda \& Thomé 2008b) }\end{array}$ & Literature & 10 & $27^{\circ} 29^{\prime} 39.12^{\prime \prime} \mathrm{S}, 58^{\circ} 44^{\prime} 38.4^{\prime \prime} \mathrm{W}$ \\
\hline O. unguis & Argentina & Resistencia, Chaco & MZSP 14748 & Museum & 10 & $27^{\circ} 29^{\prime} 39.12^{\prime \prime} \mathrm{S}, 58^{\circ} 44^{\prime} 38.4^{\prime \prime} \mathrm{W}$ \\
\hline o. convexus & Argentina & $\begin{array}{c}\text { Guaycurú Stream, } \\
\text { Chaco } \\
\end{array}$ & $\begin{array}{l}\text { CM unnumbered (Parodiz 1963), } \\
\text { (Arruda \& Thomé 2008b) }\end{array}$ & Literature & 10 & $26^{\circ} 21^{\prime} 50.4^{\prime \prime} \mathrm{S}, 60^{\circ} 51^{\prime} 16.2^{\prime \prime} \mathrm{W}$ \\
\hline O. convexus & Argentina & Navarro, Buenos Aires & $\begin{array}{c}\text { MACN } 27241 \\
\text { (Hylton-Scott \& Lapuente 1968) }\end{array}$ & Literature & 10 & $34^{\circ} 59^{\prime} 57.84^{\prime \prime S}, 59^{\circ} 16^{\prime} 42.96^{\prime \prime} \mathrm{W}$ \\
\hline
\end{tabular}


Table 3. Continued...

\begin{tabular}{|c|c|c|c|c|c|c|}
\hline Species & Country & Locality & ${ }^{1}$ Record & ${ }^{2}$ Source & $\begin{array}{c}{ }^{3} \text { Hydrographic } \\
\text { Region }\end{array}$ & Coordinates \\
\hline o. convexus & Argentina & $\begin{array}{c}\text { Las Rosas, Buenos } \\
\text { Aires }\end{array}$ & $\begin{array}{l}\text { MACN } 14860 \text { (Hylton- } \\
\text { Scott \& Lapuente 1968), } \\
\text { (Arruda \& Thomé 2008b) }\end{array}$ & Literature & 10 & $36^{\circ} 26^{\prime} 56.4^{\prime \prime} \mathrm{S}, 58^{\circ} 50^{\prime} 27.96^{\prime \prime} \mathrm{W}$ \\
\hline o. convexus & Argentina & $\begin{array}{l}\text { Chapadmalal, Buenos } \\
\text { Aires } \\
\end{array}$ & $\begin{array}{c}\text { Hylton-Scott \& Lapuente 1968; MACN } \\
27242 \text { (Arruda \& Thomé 2008b) }\end{array}$ & Literature & 11 & $38^{\circ} 54^{\prime} 43.2^{\prime \prime S}, 58^{\circ} 14^{\prime} 46.68^{\prime \prime} \mathrm{W}$ \\
\hline O. unguis & Argentina & $\begin{array}{c}\text { Barca Grande, Buenos } \\
\text { Aires }\end{array}$ & $\begin{array}{c}\text { MACN } 13996 \text { (Hylton-Scott \& } \\
\text { Lapuente 1968, Arruda \& Thomé } \\
\text { 2008b); Coscarelli \& Vidigal } 2011\end{array}$ & Literature & 10 & $34^{\circ} 14^{\prime} 54.24^{\prime \prime} \mathrm{S}, 58^{\circ} 44^{\prime} 45.6^{\prime \prime} \mathrm{W}$ \\
\hline O. unguis & Argentina & Guaycolec, Formosa & $\begin{array}{l}\text { Hylton-Scott \& Lapuente } 1968, \\
\text { Coscarelli \& Vidigal } 2011\end{array}$ & Literature & 10 & $26^{\circ} 11^{\prime} 49.2^{\prime \prime S}, 58^{\circ} 10^{\prime} 32.88^{\prime \prime} \mathrm{W}$ \\
\hline O. unguis & Argentina & Formosa, Formosa & $\begin{array}{c}\text { MLP 4567-1 (Arruda \& Thomé 2008b), } \\
\text { Coscarelli \& Vidigal } 2011\end{array}$ & Literature & 10 & $26^{\circ} 11^{\prime} 49.2^{\prime \prime S}, 58^{\circ} 10^{\prime} 32.88^{\prime \prime} \mathrm{W}$ \\
\hline O. unguis & Argentina & $\begin{array}{l}\text { Manantiales, } \\
\text { Corrientes }\end{array}$ & $\begin{array}{c}\text { Hylton-Scott \& Lapuente } 1968, \\
\text { Coscarelli \& Vidigal } 2011\end{array}$ & Literature & 10 & $27^{\circ} 55^{\prime} 19.2^{\prime \prime} \mathrm{S}, 58^{\circ} 59^{\prime} 42^{\prime \prime} \mathrm{W}$ \\
\hline O. unguis & Argentina & Oro River, Chaco & $\begin{array}{c}\text { MACN unnumbered (Hylton- } \\
\text { Scott \& Lapuente 1968), } \\
\text { Coscarelli \& Vidigal 2011 }\end{array}$ & Literature & 10 & $26^{\circ} 47^{\prime} 16.08^{\prime \prime} \mathrm{S}, 59^{\circ} 26^{\prime} 42^{\prime \prime} \mathrm{W}$ \\
\hline O. unguis & Argentina & $\begin{array}{l}\text { Tragadero River, } \\
\text { Locality between } \\
\text { Barraqueras and } \\
\text { Antequera, Chaco }\end{array}$ & Poi de Neiff et al. 1977 & Literature & 10 & $27^{\circ} 29^{\prime} 39.12^{\prime \prime} \mathrm{S}, 58^{\circ} 44^{\prime} 38.4^{\prime \prime} \mathrm{W}$ \\
\hline O. unguis & Argentina & Catamarca, Catamarca & Cazzaniga 1985 & Literature & 11 & $28^{\circ} 28^{\prime} 21^{\prime \prime} \mathrm{S}, 65^{\circ} 46^{\prime} 45.48^{\prime \prime} \mathrm{W}$ \\
\hline Omalonyx sp. & Argentina & $\begin{array}{c}\text { Ensenada, Buenos } \\
\text { Aires }\end{array}$ & MLP 31321 & Museum & 10 & $34^{\circ} 51^{\prime} 53.28^{\prime} \mathrm{S}, 57^{\circ} 54^{\prime} 30.6^{\prime \prime} \mathrm{W}$ \\
\hline Omalonyx sp. & Argentina & Atalaya, Buenos Aires & LMSM 23 & Donation & 10 & $35^{\circ} 14^{\prime} 56.4^{\prime \prime} \mathrm{S}, 57^{\circ} 31^{\prime} 53.76^{\prime \prime} \mathrm{W}$ \\
\hline Omalonyx sp. & Argentina & $\begin{array}{c}\text { Esteros de Iberá, } \\
\text { Corrientes }\end{array}$ & LMSM 16-22, 234-35 & Donation & 10 & $28^{\circ} 20^{\prime} 24^{\prime \prime} \mathrm{S}, 57^{\circ} 22^{\prime} 56.64^{\prime \prime} \mathrm{W}$ \\
\hline O. convexus & Argentina & $\begin{array}{c}\text { Ciudad Autónoma de } \\
\text { Buenos Aires, Buenos } \\
\text { Aires } \\
\end{array}$ & LMSM 3239-40; ANSP 23465 & Survey & 10 & $34^{\circ} 35^{\prime} 58.92^{\prime \prime} \mathrm{S}, 58^{\circ} 22^{\prime} 54.84^{\prime \prime} \mathrm{W}$ \\
\hline O. unguis & Argentina & Villafañe, Formosa & MLP 11878 (Arruda \& Thomé 2008b) & Literature & 10 & $26^{\circ} 14^{\prime} 10.32^{\prime \prime S}, 59^{\circ} 7 ’ 48^{\prime \prime} \mathrm{W}$ \\
\hline O. unguis & Argentina & $\begin{array}{c}\text { Bahía Blanca, Buenos } \\
\text { Aires } \\
\end{array}$ & Cazzaniga 1985 & Literature & 11 & $38^{\circ} 43^{\prime} 58.8^{\prime \prime S}, 62^{\circ} 15^{\prime} 58.68^{\prime \prime} \mathrm{W}$ \\
\hline Omalonyx sp. & Argentina & Misiones & $\begin{array}{c}\text { MLP unnumbered } \\
\text { (Gregoric et al. 2013) }\end{array}$ & Literature & 10 & $27^{\circ} 27^{\prime} 36^{\prime \prime S}, 54^{\circ} 35^{\prime} 29.4^{\prime \prime} \mathrm{W}$ \\
\hline O. unguis & Argentina & Formosa & MLP 4567-1 (Arruda \& Thomé 2008b) & Literature & 10 & $24^{\circ} 52 ’ 12 ” \mathrm{~S}, 60^{\circ} 26^{\prime} 27.6^{\prime \prime} \mathrm{W}$ \\
\hline O. unguis & Argentina & Tigre, Buenos Aires & LMSM 734 (Arruda \& Thomé 2008b) & Literature & 10 & $34^{\circ} 25^{\prime} 36.48^{\prime \prime} \mathrm{S}, 58^{\circ} 34^{\prime} 46.2^{\prime \prime} \mathrm{W}$ \\
\hline O. unguis & Argentina & $\begin{array}{c}\text { Buenos Aires, Buenos } \\
\text { Aires }\end{array}$ & ANSP 23465 & Museum & 10 & $34^{\circ} 35^{\prime} 58.92^{\prime \prime} \mathrm{S}, 58^{\circ} 22^{\prime} 54.84^{\prime \prime} \mathrm{W}$ \\
\hline $\begin{array}{l}\text { Omalonyx } \\
\text { sp.* }\end{array}$ & Chile & Juan Fernandes & Odhner, 1922, Letelier et al. 2014 & Literature & 13 & $33^{\circ} 46^{\prime} 23.52^{\prime \prime S}, 80^{\circ} 46^{\prime} 33.6^{\prime \prime} \mathrm{W}$ \\
\hline $\begin{array}{l}\text { Omalonyx } \\
\text { sp. * }\end{array}$ & Chile & Valparaiso Region & ANSP 64117 & Museum & 13 & $32^{\circ} 39^{\prime} 6.48^{\prime \prime} \mathrm{S}, 71^{\circ} 24^{\prime} 21.6^{\prime \prime} \mathrm{W}$ \\
\hline $\begin{array}{l}\text { Omalonyx } \\
\text { sp.* }\end{array}$ & Chile & $\begin{array}{c}\text { Valparaiso Region } \\
\text { Robinson Crusoe } \\
\text { Island, Juan Fernandez } \\
\text { Archipelago }\end{array}$ & ANSP 130450, A9768G & Museum & 13 & $34^{\circ} 10^{\prime} 12^{\prime \prime} \mathrm{S}, 80^{\circ} 49^{\prime} 59.88^{\prime \prime} \mathrm{W}$ \\
\hline Omalonyx sp. & Colombia & - & $\begin{array}{c}\text { FMNH unnumbered } \\
\text { (Vera-Ardila 2008) } \\
\end{array}$ & Literature & 5 & $0^{\circ} 14^{\prime} 8.88^{\prime \prime} \mathrm{S}, 72^{\circ} 30^{\prime} 0^{\prime \prime} \mathrm{W}$ \\
\hline O. matheroni & Ecuador & Limoncocha Lake & $\begin{array}{l}\text { Hermann \& Dundee 1967; FMNH } \\
\text { 157321(Tillier 1981) }\end{array}$ & Literature & 5 & $0^{\circ} 23^{\prime} 57.12^{\prime \prime} \mathrm{S}, 76^{\circ} 36^{\prime} 33.12^{\prime \prime} \mathrm{W}$ \\
\hline o. geayi & Ecuador & $\begin{array}{l}\text { Limonconcha, } \\
\text { Sucumbios }\end{array}$ & FMNH 328261 (Arruda et. al 2016) & Literature & 5 & $0^{\circ} 24^{\prime} 41.91 ” S, 76^{\circ} 37^{\prime} 31.36^{\prime \prime} \mathrm{W}$ \\
\hline O. matheroni & French Guiana & Kourou Bridge & $\begin{array}{c}\text { Tillier coll. } 4.78 \text { (Tillier 1981); MNHN } \\
\text { (Arruda \& Thomé 2008a) }\end{array}$ & Literature & 4 & $5^{\circ} 9{ }^{\prime} 39.24^{\prime \prime} \mathrm{N}, 52^{\circ} 38^{\prime} 57.48^{\prime \prime} \mathrm{W}$ \\
\hline o. geayi & French Guiana & $\begin{array}{l}\text { Route Cayene-Kourou, } \\
\text { Kaw Swamps }\end{array}$ & $\begin{array}{c}\text { MNHN, Tillier coll. 29.4.1977 } \\
\text { (Tillier 1980); MNH. (Tillier 1981), } \\
\text { (Arruda et al. 2016) }\end{array}$ & Literature & 4 & $4^{\circ} 30^{\prime} 52.92^{\prime \prime} \mathrm{N}, 52^{\circ} 39^{\prime} 57.6^{\prime \prime} \mathrm{W}$ \\
\hline
\end{tabular}


Table 3. Continued...

\begin{tabular}{|c|c|c|c|c|c|c|}
\hline Species & Country & Locality & ${ }^{1}$ Record & ${ }^{2}$ Source & $\begin{array}{c}{ }^{3} \text { Hydrographic } \\
\text { Region }\end{array}$ & Coordinates \\
\hline O. geayi & French Guiana & Kaw Swamps & LMSM 3204, 3222-24, 3226 & Survey & 4 & $4^{\circ} 30^{\prime} 52.92^{\prime \prime} \mathrm{N}, 52^{\circ} 39^{\prime} 57.6^{\prime \prime} \mathrm{W}$ \\
\hline o. matheroni & Guyana & Demerara & $\begin{array}{c}\text { Gibbson 1879; BMNH 1930.5.14.6.11 } \\
\text { (Tillier 1981) }\end{array}$ & Literature & 4 & $6^{\circ} 37^{\prime} 53.04^{\prime \prime} \mathrm{N}, 58^{\circ} 38^{\prime} 27.6^{\prime} \mathrm{W}$ \\
\hline O. matheroni & Guyana & $\begin{array}{l}\text { Manikol Swamps } \\
\text { (upper Tuyuni) }\end{array}$ & BMNH 1936.12.2.27.29 (Tillier 1981) & Literature & 4 & $5^{\circ} 57^{\prime} 18^{\prime \prime} \mathrm{N}, 57^{\circ} 41^{\prime} 26.16^{\prime \prime} \mathrm{W}$ \\
\hline Omalonyx sp. & Guyana & Georgetown & CMIOC 455 & Museum & 4 & $6^{\circ} 47^{\prime} 52.8^{\prime \prime} \mathrm{N}, 58^{\circ}$ 9'19.08”W \\
\hline O. matheroni & $\begin{array}{l}\text { Lesser Antilles, } \\
\text { Trinidad and } \\
\text { Tobago, }\end{array}$ & Port Spain & ANSP A1173 & Museum & - & $10^{\circ} 39^{\prime} 44.28^{\prime \prime} \mathrm{N}, 61^{\circ} 31^{\prime} 48^{\prime \prime} \mathrm{W}$ \\
\hline O. matheroni & $\begin{array}{l}\text { Lesser Antilles, } \\
\text { Guadeloupe }\end{array}$ & Pointe- à - Pitre & MNHN (Arruda \& Thomé, 2008a) & Literature & - & $16^{\circ} 13^{\prime} 59.88^{\prime \prime} \mathrm{S}, 61^{\circ} 31^{\prime} 59.88^{\prime \prime} \mathrm{W}$ \\
\hline O. matheroni & $\begin{array}{l}\text { Lesser Antilles, } \\
\text { Guadeloupe }\end{array}$ & Pico Spring & $\begin{array}{l}\text { LMSM 379-85, } 2186-95 \text { donated by } \\
\text { Dr. J. P. Poitier collection }\end{array}$ & Donation & - & $16^{\circ} 13^{\prime} 59.88^{\prime \prime} \mathrm{S}, 61^{\circ} 31^{\prime} 59.88^{\prime \prime} \mathrm{W}$ \\
\hline O. matheroni & $\begin{array}{l}\text { Lesser Antilles, } \\
\text { Guadeloupe }\end{array}$ & $\begin{array}{l}\text { Céligny and other } \\
\text { localities }\end{array}$ & $\begin{array}{c}\text { Lesson } 1838, \text { Tillier } 1981 \\
\text { (Dr. J. P. Poitier collection) }\end{array}$ & Literature & - & $16^{\circ} 13^{\prime} 59.88^{\prime \prime} \mathrm{S}, 61^{\circ} 31^{\prime} 59.88^{\prime \prime} \mathrm{W}$ \\
\hline o. matheroni & $\begin{array}{l}\text { Lesser Antilles, } \\
\text { Guadeloupe }\end{array}$ & Vallet Pond & ANSP A22070 & Museum & - & $16^{\circ} 13^{\prime} 59.88^{\prime \prime} \mathrm{S}, 61^{\circ} 31^{\prime} 59.88^{\prime \prime} \mathrm{W}$ \\
\hline O. matheroni & $\begin{array}{c}\text { Lesser Antilles, } \\
\text { Santa Lucia }\end{array}$ & - & Hermann \& Dundee 1967 & Literature & - & $13^{\circ} 54^{\prime} 7.2^{\prime \prime} \mathrm{N}, 60^{\circ} 58^{\prime} 42.96^{\prime \prime} \mathrm{W}$ \\
\hline o. pattersonae & $\begin{array}{l}\text { Lesser Antilles, } \\
\text { Antigua }\end{array}$ & & $\begin{array}{c}\text { Hermann \& Dundee 1967; MZUM, } \\
\text { (Patterson 1971); USNM 272284, } \\
\text { 215047; BMNH 95.1.29.17 } \\
\text { (Tillier 1981) }\end{array}$ & Literature & - & $17^{\circ} 42^{\prime} 14.4^{\prime \prime} \mathrm{N}, 61^{\circ} 48^{\prime} 25.2^{\prime \prime} \mathrm{W}$ \\
\hline O. unguis & $\begin{array}{l}\text { Lesser Antilles, } \\
\text { Antigua and } \\
\text { Barbuda }\end{array}$ & & ANSP 71975 & Museum & - & $17^{\circ} 42^{\prime} 14.4^{\prime \prime} \mathrm{N}, 61^{\circ} 48^{\prime} 25.2^{\prime \prime} \mathrm{W}$ \\
\hline o. matheroni & $\begin{array}{c}\text { Lesser Antilles, } \\
\text { Trinidad and } \\
\text { Tobago }\end{array}$ & & USNM 162058 & Museum & - & $10^{\circ} 26^{\prime} 44.52^{\prime \prime} \mathrm{N}, 61^{\circ} 16^{\prime} 17.04^{\prime \prime} \mathrm{W}$ \\
\hline o. matheroni & $\begin{array}{l}\text { Lesser Antilles, } \\
\text { Trinidad }\end{array}$ & & $\begin{array}{c}\text { BMNH 1950.618.407; LMNH } \\
\text { (Tillier 1980, 1981) }\end{array}$ & Literature & - & $10^{\circ} 26^{\prime} 44.52^{\prime \prime} \mathrm{N}, 61^{\circ} 16^{\prime} 17.04^{\prime \prime} \mathrm{W}$ \\
\hline O. matheroni & $\begin{array}{l}\text { Lesser Antilles, } \\
\text { Trinidad and } \\
\text { Tobago }\end{array}$ & $\begin{array}{l}\text { Tunapuna-Piarco, } \\
\text { Saint George East }\end{array}$ & USDA 100453 (Arruda et al. 2016) & Literature & & $\begin{array}{c}10^{\circ} 37^{\prime} 29.7336^{\prime \prime} \mathrm{N}, 61^{\circ} \\
13^{\prime} 6.5316^{\prime \prime} \mathrm{W}\end{array}$ \\
\hline O. matheroni & $\begin{array}{c}\text { Lesser Antilles, } \\
\text { Monserrat }\end{array}$ & St. Peter & ANSP A23921 & Museum & - & $16^{\circ} 44^{\prime} 18.24^{\prime \prime} \mathrm{N}, 62^{\circ} 12^{\prime} 31.68^{\prime \prime} \mathrm{W}$ \\
\hline Omalonyx sp. & $\begin{array}{l}\text { Lesser Antilles, } \\
\text { Antigua and } \\
\text { Barbuda }\end{array}$ & (2) & USNM 460663, 151347 & Museum & - & $17^{\circ} 42^{\prime} 14.4^{\prime \prime} \mathrm{N}, 61^{\circ} 48^{\prime} 25.2^{\prime \prime} \mathrm{W}$ \\
\hline O. unguis & Paraguay & $\begin{array}{c}\text { Paraná River near } \\
\text { Corrientes and Moxos } \\
\text { (Bolivia) }\end{array}$ & d'Orbigny 1835, 1837; Tillier (1981) & Literature & 10 & $27^{\circ} 57^{\prime} 57.6 ” S, 58^{\circ} 14^{\prime} 35.52^{\prime \prime} \mathrm{W}$ \\
\hline O. unguis & Paraguay & Asunción & $\begin{array}{c}\text { CM unnumber (Parodiz 1963); MACN } \\
19968 \text { (Arruda \& Thomé 2008b) }\end{array}$ & Literature & 10 & $25^{\circ} 16^{\prime} 32.88^{\prime \prime} \mathrm{S}, 57^{\circ} 38^{\prime} 16.08^{\prime \prime} \mathrm{W}$ \\
\hline O. unguis & Paraguay & Asunción & LMSM 3436 & Survey & 10 & $25^{\circ} 16^{\prime} 32.88^{\prime \prime} \mathrm{S}, 57^{\circ} 38^{\prime} 16.08^{\prime \prime} \mathrm{W}$ \\
\hline O. unguis & Paraguay & Pilar, Ne & LMSM 3341 & Survey & 10 & $26^{\circ} 51^{\prime} 11.88^{\prime \prime} \mathrm{S}, 58^{\circ} 17^{\prime} 44.88^{\prime \prime} \mathrm{W}$ \\
\hline O. convexus & Paraguay & San Bernardino & CM unumber (Parodiz 1963) & Literature & 10 & $25^{\circ} 16^{\prime} 46.92^{\prime \prime} \mathrm{S}, 57^{\circ} 17^{\prime} 7.08^{\prime \prime} \mathrm{W}$ \\
\hline O. unguis & Paraguay & Villa Rica & $\begin{array}{l}\text { MCZ unumber (Parodiz 1963), } \\
\text { (Arruda \& Thomé 2008b) }\end{array}$ & Literature & 10 & $25^{\circ} 49^{\prime} 26.4^{\prime \prime S}, 56^{\circ} 24^{\prime} 18^{\prime \prime} \mathrm{W}$ \\
\hline O. unguis & Paraguay & Puerto Guaraní & $\begin{array}{c}\text { MACN 18181 } \\
\text { (Hylton-Scott \& Lapuente 1968), } \\
\text { Coscarelli \& Vidigal } 2011 \\
\end{array}$ & Literature & 10 & $21^{\circ} 17^{\prime} 20.4^{\prime \prime} \mathrm{S}, 57^{\circ} 55^{\prime} 26.4^{\prime \prime} \mathrm{W}$ \\
\hline Omalonyx sp. & Paraguay & $\begin{array}{l}\text { Riacho Nepengue, } \\
\text { Concepción }\end{array}$ & INPA 485 & Museum & 10 & $22^{\circ} 27^{\prime} 23.76^{\prime \prime} \mathrm{S}, 57^{\circ} 35^{\prime} 19.68^{\prime \prime} \mathrm{W}$ \\
\hline O. unguis & Paraguay & Paso de Patria, $\tilde{\mathrm{Ne}}$ & LMSM 3434,3350 & Survey & 10 & $27^{\circ} 14^{\prime} 52.08^{\prime \prime} \mathrm{S}, 58^{\circ} 32^{\prime} 20.04^{\prime \prime} \mathrm{W}$ \\
\hline Omalonyx sp. & Peru & $\begin{array}{l}\text { Tarapoto, San Martín } \\
\text { Morales, San Martín }\end{array}$ & $\begin{array}{c}\text { MZSP 43266; USMN } 612118 \\
\text { (Tillier 1981) }\end{array}$ & Literature & 5 & $6^{\circ} 11^{\prime} 6.36^{\prime \prime} \mathrm{S}, 76^{\circ} 28^{\prime} 44.4^{\prime \prime} \mathrm{W}$ \\
\hline O. matheroni & Peru & San Martín & MCP 08850 & Museum & 5 & $6^{\circ} 25^{\prime} 30.72^{\prime \prime} \mathrm{S}, 76^{\circ} 49^{\prime} 51.24^{\prime \prime} \mathrm{W}$ \\
\hline
\end{tabular}


Table 3. Continued...

\begin{tabular}{|c|c|c|c|c|c|c|}
\hline Species & Country & Locality & ${ }^{1}$ Record & ${ }^{2}$ Source & $\begin{array}{l}{ }^{3} \text { Hydrographic } \\
\text { Region }\end{array}$ & Coordinates \\
\hline o. unguis & Peru & $\begin{array}{c}\text { Lake Yarinacocha, } \\
\text { Ucayali }\end{array}$ & Ramirez, 1991, Ramirez et al. 2003 & Literature & 5 & $8^{\circ} 18^{\prime} 29.88^{\prime \prime S}, 74^{\circ} 36^{\prime} 7.2^{\prime \prime} \mathrm{W}$ \\
\hline O. matheroni & Suriname & Zanderij & RMNH 15.6.67 (Tillier, 1981) & Literature & 4 & $5^{\circ} 27^{\prime} 21.24^{\prime \prime} \mathrm{N}, 55^{\circ} 12^{\prime} 39.6^{\prime \prime} \mathrm{W}$ \\
\hline O. matheroni & Suriname & Belwaarde & RMNH 2.9.69 (Tillier 1981) & Literature & 4 & $5^{\circ} 51^{\prime} 6.12^{\prime \prime} \mathrm{N}, 55^{\circ} 52^{\prime} 55.2^{\prime \prime} \mathrm{W}$ \\
\hline O. matheroni & Suriname & Paramaribo & RMNH 6.68 (Tillier 1981) & Literature & 4 & $5^{\circ} 51^{\prime} 7.2^{\prime \prime} \mathrm{N}, 55^{\circ} 12^{\prime} 13.32^{\prime \prime} \mathrm{W}$ \\
\hline O. geayi & Suriname & Paramaribo & FLMNH 463461 (Arruda et al. 2016) & Literature & 4 & $5^{\circ} 51^{\prime} 7.2^{\prime \prime} \mathrm{N}, 55^{\circ} 12^{\prime} 13.32^{\prime \prime} \mathrm{W}$ \\
\hline O. matheroni & Suriname & $\begin{array}{l}\text { Brokobakka, } \\
\text { Brokopondo }\end{array}$ & RMNH unnumbered (Tillier 1981) & Literature & 4 & $3^{\circ} 53^{\prime} 22.56^{\prime \prime} \mathrm{N}, 55^{\circ} 34^{\prime} 28.56^{\prime \prime} \mathrm{W}$ \\
\hline O. unguis & Uruguay & $\begin{array}{l}\text { Fuente Salto, close to } \\
\text { Salto city }\end{array}$ & Olazarri 1979 & Literature & 10 & $31^{\circ} 23^{\prime} 31.2^{\prime \prime} \mathrm{S}, 57^{\circ} 49^{\prime} 55.2^{\prime \prime} \mathrm{W}$ \\
\hline Omalonyx sp. & Uruguay & Montevideo & MZSP 31873 & Museum & 10 & $34^{\circ} 54^{\prime} 43.2^{\prime \prime} \mathrm{S}, 56^{\circ} 9^{\prime} 52.2^{\prime \prime} \mathrm{W}$ \\
\hline O. convexus & Uruguay & Montevideo & ANSP 70261 & Museum & 10 & $34^{\circ} 54^{\prime} 43.2^{\prime \prime S}, 56^{\circ} 9^{\prime} 52.2^{\prime \prime} \mathrm{W}$ \\
\hline O. unguis & Uruguay & $\begin{array}{l}\text { Near from Carrasco } \\
\text { Stream, Montevideo }\end{array}$ & Scarabino 2003 & Literature & 10 & $34^{\circ} 54^{\prime} 43.2^{\prime \prime} \mathrm{S}, 56^{\circ} 9^{\prime} 52.2^{\prime \prime} \mathrm{W}$ \\
\hline o. convexus & Uruguay & $\begin{array}{l}\text { Miguelete Stream, } \\
\text { Prado, Montevideo }\end{array}$ & Scarabino 2003 & Literature & 10 & $34^{\circ} 54^{\prime} 43.2^{\prime \prime} \mathrm{S}, 56^{\circ} 9^{\prime} 52.2^{\prime \prime} \mathrm{W}$ \\
\hline o. convexus & Uruguay & Canelones & $\begin{array}{c}\text { MNHNM8691 } \\
\text { (Arruda \& Thomé 2008b) }\end{array}$ & Literature & 10 & $34^{\circ} 43^{\prime} 29.28^{\prime \prime} \mathrm{S}, 55^{\circ} 57^{\prime} 34.56^{\prime \prime} \mathrm{W}$ \\
\hline O. unguis & Uruguay & $\begin{array}{l}\text { Colonia, Uruguai } \\
\text { River in front of Punta } \\
\text { Gorda }\end{array}$ & $\begin{array}{l}\text { MNHNM } 3368 \\
\text { (Arruda \& Thomé 2008b) }\end{array}$ & Literature & 10 & $33^{\circ} 57^{\prime} 7.56^{\prime \prime} \mathrm{S}, 58^{\circ} 21^{\prime} 36^{\prime \prime} \mathrm{W}$ \\
\hline O. matheroni & Venezuela & Caracas & $\begin{array}{l}\text { Martens 1873, Tillier 1981, } \\
\text { Arruda et al. } 2009\end{array}$ & Literature & 2 & $10^{\circ} 28^{\prime} 43.68^{\prime \prime} \mathrm{N}, 66^{\circ} 54^{\prime} 12.24^{\prime \prime} \mathrm{W}$ \\
\hline O. matheroni & Venezuela & $\begin{array}{l}\text { Laguna de Ramón } \\
\text { Coronel, near Bejuma }\end{array}$ & Baker 1925, 1926; Martinez 1993 & Literature & 3 & $9^{\circ} 50^{\prime} 47.4^{\prime \prime} \mathrm{N}, 68^{\circ} 12^{\prime} 52.92^{\prime \prime} \mathrm{W}$ \\
\hline O. pattersonae & Venezuela & $\begin{array}{c}\text { Lake Valencia, } \\
\text { Carabobo }\end{array}$ & Martinez 1993 & Literature & 2 & $10^{\circ} 9^{\prime} 13.32^{\prime \prime} \mathrm{N}, 67^{\circ} 53^{\prime} 40.92^{\prime \prime} \mathrm{W}$ \\
\hline O. pattersonae & Venezuela & Zuata Dam, Aragua & Martinez 1993 & Literature & 2 & $10^{\circ} 11^{\prime} 39.84^{\prime \prime} \mathrm{N}, 67^{\circ} 23^{\prime} 40.56^{\prime \prime} \mathrm{W}$ \\
\hline O. matheroni & Venezuela & $\begin{array}{l}\text { Santa Elena de } \\
\text { Uiarém, Bolívar }\end{array}$ & $\begin{array}{c}\text { LMSM 2271-75, 2336-372353-54, } \\
2356-57,2372-73,2377,2378-79 \\
2522-24\end{array}$ & Survey & 5 & $4^{\circ} 35^{\prime} 43.08^{\prime \prime} \mathrm{N}, 61^{\circ} 6^{\prime} 10.08^{\prime \prime} \mathrm{W}$ \\
\hline O. pattersonae & Venezuela & Lake Tacarigua & ANSP 161142 & Museum & 2 & $10^{\circ} 48^{\prime} 10.8^{\prime \prime} \mathrm{N}, 65^{\circ} 48^{\prime} 21.24^{\prime \prime} \mathrm{W}$ \\
\hline O. pattersonae & Venezuela & - & ANSP 140961 & Museum & - & unespecified locality \\
\hline Omalonyx sp. & Venezuela & Amacuro Delta & MCP 09193 & Museum & 2 & $9^{\circ} 29^{\prime} 31.2^{\prime \prime} \mathrm{N}, 62^{\circ} 31^{\prime} 22.8^{\prime \prime} \mathrm{W}$ \\
\hline O. unguis & Bolivia & Santa Cruz de la Sierra & MZUM unnumbered (Parodiz 1963) & Literature/ & 5 & $17^{\circ} 7^{\prime} 59.88^{\prime \prime S}, 63^{\circ} 55^{\prime} 12^{\prime \prime W}$ \\
\hline O. pattersonae & Bolivia & Santa Cruz de la Sierra & MCP 09185 & Museum & 5 & $17^{\circ} 7 ' 59.88^{\prime \prime S}, 63^{\circ} 55^{\prime} 12^{\prime \prime W}$ \\
\hline o. geayi & Bolivia & Santa Cruz & MNKMO 7 (Arruda et al. 2016 & Literature & 10 & $\begin{array}{c}19^{\circ} 7^{\prime} 45.0912^{\prime \prime} \mathrm{S}, 61^{\circ} \\
41^{\prime} 57.804 ’ \mathrm{~W}\end{array}$ \\
\hline Omalonyx sp. & Bolivia & - & ANSP A1826c & Museum & 5 & $17^{\circ} 7^{\prime} 59.88^{\prime \prime} \mathrm{S}, 63^{\circ} 55^{\prime} 12^{\prime \prime} \mathrm{W}$ \\
\hline o. unguis & $\begin{array}{c}\text { Lesser Antilles, } \\
\text { Guadeloupe }\end{array}$ & & ANSP 23463 & Museum & - & $16^{\circ} 15^{\prime} 47.52^{\prime \prime} \mathrm{N}, 61^{\circ} 33^{\prime} 10.8^{\prime \prime} \mathrm{W}$ \\
\hline O. matheroni & $\begin{array}{l}\text { Lesser Antilles, } \\
\text { Montserrat }\end{array}$ & Daly River, Saint Peter & ANSP A23919 & Museum & - & $16^{\circ} 44^{\prime} 18.24^{\prime \prime} \mathrm{N}, 62^{\circ} 12^{\prime} 31.68^{\prime \prime} \mathrm{W}$ \\
\hline O. matheroni & $\begin{array}{l}\text { Lesser Antilles, } \\
\text { Montserrat }\end{array}$ & & ANSP 144327 & Museum & - & $16^{\circ} 44^{\prime} 13.92^{\prime \prime} \mathrm{N}, 62^{\circ} 11^{\prime} 33.36^{\prime \prime} \mathrm{W}$ \\
\hline O. matheroni & $\begin{array}{l}\text { Lesser Antilles, } \\
\text { Monserrat }\end{array}$ & $\begin{array}{l}\text { Runaway Ghaut, } \\
\text { Saint Peter }\end{array}$ & $\begin{array}{c}\text { ANSP A23917-18, A23920, 466625, } \\
\text { A23921 }\end{array}$ & Museum & - & $16^{\circ} 45^{\prime} 27^{\prime \prime} \mathrm{N}, 62^{\circ} 13^{\prime} 12^{\prime \prime} \mathrm{W}$ \\
\hline O. matheroni & $\begin{array}{l}\text { Lesser Antilles, } \\
\text { Montserrat }\end{array}$ & Woollands, Saint Peter & ANSP A23921 & Museum & - & $16^{\circ} 44^{\prime} 18.24^{\prime \prime} \mathrm{N}, 62^{\circ} 12^{\prime} 31.68^{\prime \prime} \mathrm{W}$ \\
\hline
\end{tabular}

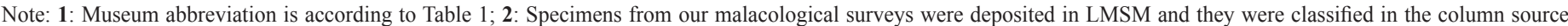

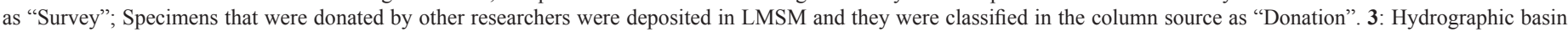

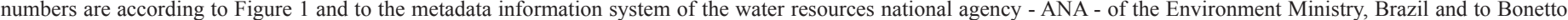
1994); * Specimens from Chile that were previously identified as O. gayana, however, Tillier 1981 considered that this species belongs to the genus Succinea. 


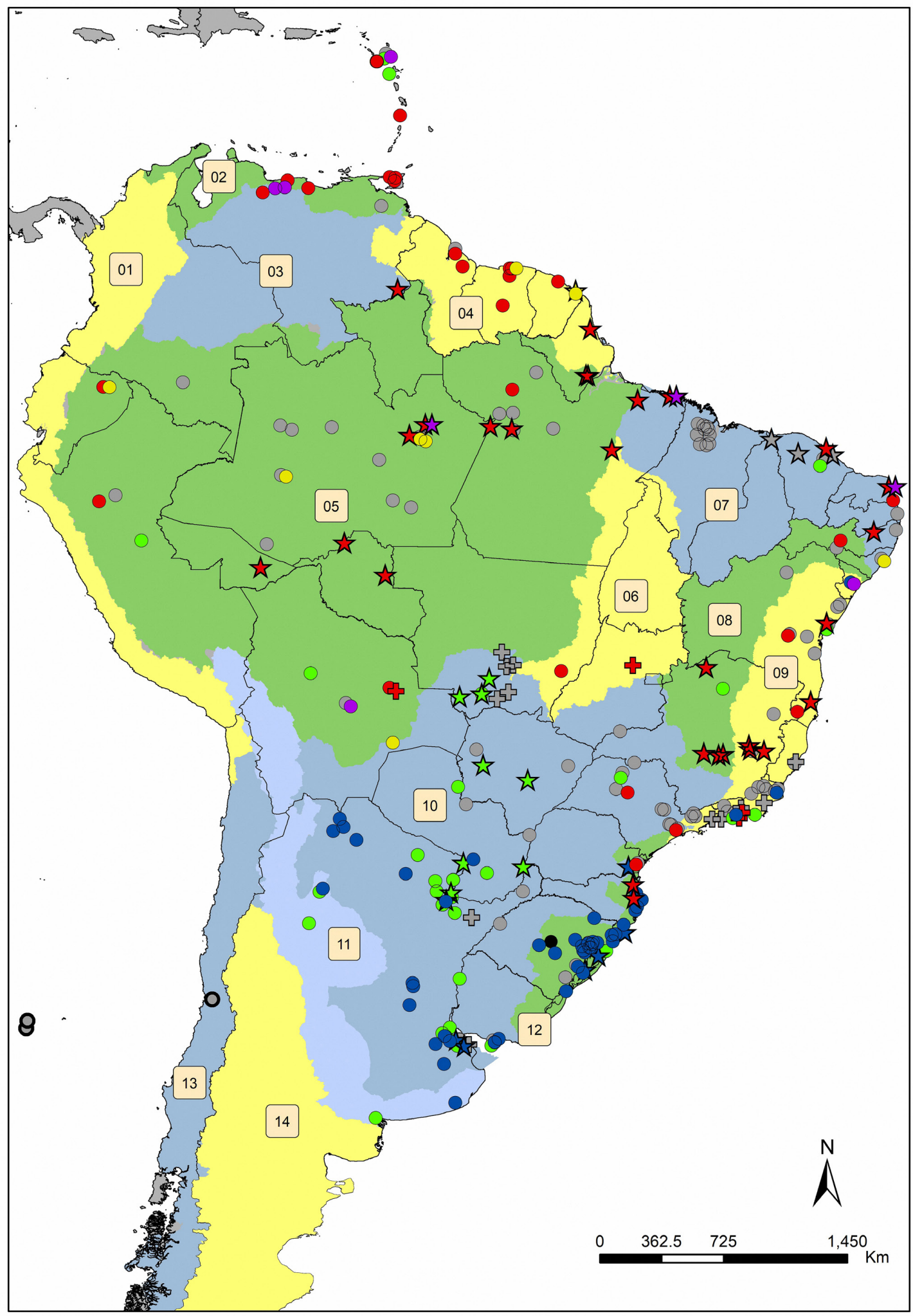

Figure 1. Updated distribution of the genus Omalonyx across Neotropical region. The coloured areas represent different hydrographic regions and their respective numbers. The solid black line indicates the borders among South American countries and within Brazilian administrative regions. The species records were represented by colours dots: red $=O$. matheroni; blue $=O$. convexus; green $=O$. unguis; yellow $=O$. geayi, purple $=O$. pattersonae, black $=O$. brasiliensis; gray $=$ do not identified until species level, or Omalonyx sp. and gray circle with black border $=$ Specimens from Chile that were previously identified as $O$. gayana. The stars represents the records made in our surveys, the circles represents literature or museums data and the cross symbol represents the samples that were donated by other researchers. Details about each sample are on tables 2 and 3. Hydrographic regions are indicated by numbers: 1) Pacific Coast; 2) Caribbean sea; 3) Orinoco River; 4) Amapá-Esequibo; 5) Amazon River; 6) Tocantins River; 7) North Atlantic; 8) São Francisco River; 9) Eastern Atlantic; 10) Del Plata; 11) Border strip of the Brasilica (North) and the Chilean-Patagonian (South West) Subregion; 12) Eastern; 13) Chilean-Patagonian Subregion of the Pacific Versant; 14) Chilean-Patagonian Subregion of the Atlantic Versant. 
Table 4. Bioclimatic variables used in models development.

\begin{tabular}{cc}
\hline Variable & Description \\
\hline Alt & Altitude \\
bio1 & Annual mean temperature \\
bio2 & Mean diurnal range (monthly mean, $\mathrm{T}^{\circ}$ max- $\mathrm{T}^{\circ}$ min) \\
bio3 & Isothermality (bio2/bio7) $\mathrm{x} 100$ \\
bio4 & Temperature seasonality (standard deviation $\mathrm{x}$ 100) \\
bio5 & Maximum temperature of warmest month \\
bio6 & Minimum temperature of coldest month \\
bio7 & Temperature annual range (bio5-bio6) \\
bio8 & Mean temperature of wettest quarter \\
bio9 & Mean temperature of driest quarter \\
bio10 & Mean temperature of the warmest quarter \\
bio11 & Mean temperature of coldest quarter \\
bio12 & Annual precipitation \\
bio13 & Precipitation of wettest month \\
bio14 & Precipitation of driest month \\
bio15 & Precipitation seasonality (coefficient of variation) \\
bio16 & Precipitation of wettest quarter \\
bio17 & Precipitation of driest quarter \\
bio18 & Precipitation of the warmest quarter \\
bio19 & Precipitation of the coldest quarter \\
\hline
\end{tabular}

To minimize the spatial autocorrelation of the bioclimatic variables and to determine which variables contributed more to the development of the model, a principal component analysis (PCA) was carried out in the areas of Omalonyx species occurrence. The resulting rasters were used to generate a potential distribution model for South America. The input parameters followed the MaxEnt (version 3.3.3k) (Phillips et al. 2004) default options except for the threshold rule, which was set as "minimum training presence". The resulting models were processed and reclassified using ArcGIS. A binary map (absence-presence) for the potential distribution of a species was generated considering the average map that represents the induced and adjusted habitat of each species.

\section{Results}

\section{Literature review}

Inferences on the distribution of Omalonyx species have been made since its description, and it was assumed to be throughout South America east of the Andes (d'Orbigny 1837). This first inference was based on records from Bolivia and Paraguay (d'Orbigny 1835a, 1837). However, at that time (1837), the name Omalonyx was used as the subgenus Succinea (Omalonyx) unguis. Later, Omalonyx was used at the genus level (Herrmannsen 1849). In addition to the publications of d'Orbigny other species were described at that time as showed below. In the next century, Patterson (1971) extended the distribution of the genus to several West Indies islands and Central America. However, the basis for this conclusion was not clearly demonstrated. After that, Tillier (1981) demonstrated the occurrence of Omalonyx species in all South America east of the Andes and the Lesser Antilles. His conclusion was based on preserved specimens from malacological collections that did not include significant sampling material from Brazil, although there were already records on the presence of Omalonyx spp. in the country years before (e.g., Moricand 1836, Gibbons 1879). In fact, Tillier (1981) studied animals from only eight localities in Brazil, and four of these could not be clearly located and were excluded from his study. In this work, Tillier also discusses the origin and evolution of South American and Juan Fernandéz Islands (Chile) succineids. He suggests that the southeast region of Brazil is the center of Omalonyx radiation once three species can be found in this region (O. unguis, O. matheroni and $O$. brasiliensis). Details on the records of Omalonyx occurrence in the Neotropics are given below in the subsections "Omalonyx in Brazil" and "Omalonyx in other South American countries and Lesser Antilles".

\subsection{Literature review: Omalonyx in Brazil}

The first record of Omalonyx in Brazil was made in the $19^{\text {th }}$ century in the Northeastern region (Moricand 1836) on a lake in Bahia State named "la Digue" or "le Baril". The specimens were identified as O. unguis. Hidalgo (1869) also identified as $O$. unguis specimens collected by Paz and Martinez in Bahia State. Two years later, the same author (Hidalgo 1872) stated that Paz and Martinez collected those specimens at "Lago Dique" (Dike Lake), Salvador, Bahia. We suppose that the specimens cited by Moricand (1836) and Hidalgo $(1869,1870)$ were collected in an urban lake known since the colonial period as "Dique do Tororó". Gibbons (1879) identified two different species of Omalonyx in Bahia State, $O$. unguis and $O$. matheroni. However, no other information about the locality was provided, and the morphology described in Gibbons (1879) is not compatible with $O$. unguis; thus these records were not included in Table 2. Omalonyx convexus was described by Martens (1968) as Succinea convexa from specimens collected in Porto Alegre, Rio Grande do Sul State, in southern Brazil (type locality), and it was later included in the genus Omalonyx (Arruda \& Thomé 2008b). The map provided by Arruda and Thomé (2008b) shows overlapping distribution of $O$. convexus and O. unguis in the Argentinean region of the Paraná River basin (in Figure 1 the Paraná River basin is included in the Del Plata hydrographic region) and adjacent localities in Uruguay. Omalonyx brasiliensis was described by Simroth (1896) based on specimens collected by H. von Ihering in Rio Grande do Sul State.

In the $20^{\text {th }}$ century, specimens of Omalonyx infected by Leucochloridium, an avian trematode, were found by Lutz (1921) in the surroundings of the institute where he used to work in Rio de Janeiro city, Rio de Janeiro State. The author also reported that this slug is abundant in the north of Brazil. This trematode was also found in birds from Mato Grosso and used in the experimental infection of Omalonyx (Travassos 1928). Both of these works have a parasitological focus, and the specific identification of the slug was not provided. Haas (1939) registered O. unguis for the State of Pernambuco but did not specify the locality. Parodiz (1957) recorded O. unguis in Brazil but did not include the locality. He also mentioned the occurrence of O. unguis in Porto Alegre (Rio Grande do Sul State) and observed its similarity to $O$. convexus. Based on specimens from the MCZ (lot number unavailable), Parodiz (1963) registered the occurrence of Omalonyx in Rio de Janeiro (O. unguis) and Camaquã, Rio Grande do Sul. The specimens from Camaquã were later identified as $O$. convexus (Arruda \& Thomé 2008b). Lange de Morretes (1949) recorded O. brasiliensis and O. unguis in Vila Nova (Bahia State) and O. matheroni in Santarém (Pará State). In a taxonomical review using specimens from institutional collections, Tillier (1981) studied animals from eight localities in Brazil. Three of them were easily identified: Santarém and Alenquer in Pará State, and Rio de Janeiro in Rio de Janeiro State. The other four localities, due to insufficient labeling information, could not be precisely located. Two of them were only superficially specified: "Brazil" Pernambuco State and Praya (=Praia) Grande (Rio de Janeiro State). The other two localities could not be identified: Santa Amélia, Amazonas and "Makthlawara" (BMNH 1929.10.2.6.22). This author also mentioned the occurrence of Omalonyx in Porto Alegre, Rio Grande do Sul State, and cited Martens (1868) and Simroth (1896), who also recorded O. brasiliensis in this State (Tillier 1981). The distribution of Omalonyx in Brazil was revisited by two authors whose results were similar to those of Lange de Morretes (1949). First, Salgado \& Coelho (2003) reported some species of the 
genus in a review about Brazilian land snails but did not cite any locality. After that, Simone (2006) cited the occurrence of O. brasiliensis in Rio Grande do Sul State and considered the occurrence area for the genus Omalonyx to be from Bolivia to Patagonia. Several other records were made in Brazil, including Minas Gerais (Oliveira \& Almeida 2000, Arruda et al. 2006, Montresor et al. 2008) and São Paulo State (Arruda et al. 2009, Eduardo et al. 2012) in the southeast; Paraná (Arruda et al. 2009, Coscarelli \& Vidigal 2011), Rio Grande do Sul (Arruda \& Thomé 2008a, b, Arruda \& Thomé 2011, Maltchik et al. 2010) and Santa Catarina State (Agudo-Padron 2008, 2012) in the south; Mato Grosso and Mato Grosso do Sul State (Coscarelli \& Vidigal 2011) in the central west; Pernambuco (Dutra-Clarke et al. 2001), Sergipe (Jesus \& Manso, 2010) and Maranhão State (Cantanhede et al. 2014) in the northeast; and Amazonas State in the North (Pimpão 2007, Garcia et al. 2012). Coscarelli \& Vidigal (2011) investigated the distribution of $O$. unguis in Brazil and included new records for this species. Recently, new records for $O$. geayi extended the distribution range of this species in northern South America including Brazil (Arruda et al. 2016). The presence of Omalonyx was recorded in several Brazilian States representing all geographic regions (Table 2). All data and details (i.e., species and coordinates) on species distribution in Brazil were included in Table 2 and are shown in Figure 1.

\subsection{Literature review: Omalonyx in other South American countries and Lesser Antilles}

Several South American and Lesser Antilles countries are included in the distribution area of the genus Omalonyx (Figure 1, Table 3). The occurrence of O. unguis in Paraguay and Bolivia was reported by d'Orbigny (1835a, b, 1837). On this occasion, the author did a complete textual description of the external appearance of the animal and specified the collection sites: the flooded margins of Paraná River, near Corrientes, and the swamps of Moxos Province in Bolivia. Corrientes is a city on the riverbank of the Paraná River in Argentina, very close to the frontier with Paraguay, which is defined by the Paraná River itself. Later, Asunción, in Paraguay, was considered to be the type locality for O. unguis (Arruda \& Thomé 2008b).

Omalonyx matheroni was described from individuals collected in Guadeloupe, Lesser Antilles (Potiez \& Michaud 1835). Tillier (1981) studied specimens he collected or borrowed from institutional collections that were from several localities including the Lesser Antilles, Guiana, Suriname, French Guyana, Ecuador, Paraguay, Argentina and Peru (Table 3). Omalonyx occurrence in South American countries has been well documented by several authors: Argentina (Arruda \& Thomé 2008a, b); Brazil (see above the topic geographic distribution of Omalonyx in Brazil); Colombia (Vera-Ardila 2008); Uruguay (Olazarri 1979, Scarabino 2003, Arruda \& Thomé 2008b.); French Guiana (Tillier 1981, Arruda et al. 2016); Ecuador (Hermann \& Dundee 1967, Tillier 1981, Arruda et al. 2016); Suriname (Tillier 1981, Arruda et al. 2016) Venezuela (Martens 1873, Baker 1925, 1926, Escarbassiere 1993); Guyana (Gibbons 1879, Tillier 1981); Peru (Tillier 1981, Ramirez, 1991; Ramirez et al. 2003) and Paraguay (Hylton-Scott \& Lapuente 1968). Parodiz (1963) listed Omalonyx specimens from Paraguay, Argentina and Bolivia that were deposited in different institutional collections. Cazzaniga (1985), based on a bibliographic review, concluded that the known distribution area for $O$. unguis is southern Brazil, northern and eastern Argentina, Uruguay, Paraguay, and Bolivia and that the presence of $O$. unguis in the west of Argentina is known only in Tucumám (Hylton-Scott 1971) and Catamarca Provinces. The latter represents a new record made by Cazzaniga (1985) himself. Arruda \& Thomé (2008b) showed that the distributions of $O$. convexus and $O$. unguis overlap in the Argentinean area of the Paraná River hydrographic region and adjacent localities in Uruguay. Gutiérrez Gregoric et al. (2013) recorded the occurrence of Omalonyx sp. in Misiones Province, Argentina using data from the La Plata Museum Mollusk Collection, literature and his own field work. For further literature data about Omalonyx occurrence in Argentina see Table 3. There are some records of Omalonyx in Chile (Odhner 1922, Letellier et al. 2003, 2014), and some of them are recent. However, in 1981, Tillier studied specimens from Chile that were identified as $O$. gayana and stated that, despite its limaciform shape, the species belongs to the genus Succinea. The recent records from Chile (Letellier et al. 2003, 2014) do not include a description of the specimens nor any discussion on this taxonomic issue related to Chilean succineids. Thus, we suppose that the specimens are in fact Succinea gayana.

The distribution of the genus in the Lesser Antilles is also well documented. Succinea haliotidea Mittre 1841 was found in Martinique by Mittre (Tillier 1981). However, Tillier (1981) did not include this species in the genus Omalonyx, and indicates that they do not even belong to the family Succineidae once neither he nor Fischer found any Omalonyx in Martinique. Therefore, we did not include Martinique within the occurrence area of Omalonyx. Hermann \& Dundee (1967) studied Omalonyx from Sta. Lucia and Antigua, both in the Lesser Antilles. Other authors have also worked in this region. Patterson (1971) and Tillier (1981) studied specimens from Antigua, and Robinson et al. (2009) recorded the occurrence of Omalonyx in several localities in Dominica. Arruda et al. (2016) showed new records of $O$. geayi and extended the range of distribution of this species in South America to include Suriname, Ecuador, and Bolivia. All references and localities are in Table 3.

\section{Records from institutional collections}

Based on data of Brazilian institutional collections obtained through literature, museum databases or onsite research, we verified 21 records for the genus in the North region of the country, 28 in the Northeast, four in the Central West, 33 in the Southeast and 31 in the South (Table 2). Specimens were found in eight Brazilian institutional collections (INPA, MCP, MCNZ, MZSP, MNRJ, UFS, ZUECA and CMIOC). Among the institutional collections that were visited, two of them did not present specimens belonging to the genus (MIRR and MPEG) in (Table 1). Brazilian specimens of Omalonyx were also registered in six foreign institutional collections (ANSP, FMNH, $\mathrm{BMNH}, \mathrm{ZMB}, \mathrm{MNHN}$, and MCZ). We also investigated the presence of Omalonyx in other South American countries and Lesser Antilles using engines for virtual searching in databases from Brazilian (CMIOC, MCP, MZSP, MNRJ) and foreign institutional collections (ANSP, BMNH, USNM, CM, MACN, MLP) (Table 3).

\section{Malacological surveys in Brazil}

Specimens of Omalonyx were sampled across Brazilian hydrographic regions, and the sites were classified above according to the presence or absence of Omalonyx spp. (Table 2 and 5).

\subsection{Surveyed sites with Omalonyx spp. presence}

Specimens were collected in all Brazilian geographic regions, and we found Omalonyx in three States of the South region (Rio Grande do Sul, Santa Catarina, Paraná) where six localities were sampled, one State in the Southeast region (Minas Gerais) where seven localities were sampled, two States in the Central-west region (Mato Grosso, Mato Grosso do Sul) where four localities were sampled, five States in the Northeast region (Piauí, Ceará, Rio Grande do Norte, Pernambuco and Bahia) where nine localities were sampled, and five States (Acre, Amazonas, Pará, Rondônia and Amapá) in the North region where 15 localities were sampled. Among these localities, we made new records for four Brazilian States: Acre, Rondônia, Amapá and Piauí. All coordinates for 41 surveyed sites are listed in Table 2 and indicated in the map (Figure 1). 
Table 5. Surveyed sites within the Brazilian administrative regions where Omalonyx spp. was absent.

\begin{tabular}{|c|c|c|}
\hline $\begin{array}{c}\text { Administrative } \\
\text { regions }\end{array}$ & Locality & Coordinates \\
\hline \multirow{5}{*}{ Distrito Federal } & \multirow{4}{*}{ Brasília } & $15^{\circ} 51^{\prime} 36^{\prime \prime} \mathrm{S}, 47^{\circ} 52^{\prime} 20^{\prime \prime} \mathrm{W}$ \\
\hline & & $15^{\circ} 50^{\prime} 21^{\prime \prime} \mathrm{S}, 47^{\circ} 54^{\prime} 26^{\prime \prime} \mathrm{W}$ \\
\hline & & $15^{\circ} 50^{\prime} 41^{\prime \prime} \mathrm{S}, 47^{\circ} 54^{\prime} 59^{\prime \prime} \mathrm{W}$ \\
\hline & & $15^{\circ} 44^{\prime} 26^{\prime \prime} \mathrm{S}, 47^{\circ} 52^{\prime} 52^{\prime \prime} \mathrm{W}$ \\
\hline & Brazlândia & $15^{\circ} 42^{\prime} 49^{\prime \prime} \mathrm{S}, 48^{\circ} 12^{\prime} 14^{\prime \prime} \mathrm{W}$ \\
\hline \multirow{8}{*}{ Tocantins } & \multirow{3}{*}{ Palmas } & $10^{\circ} 12^{\prime} 34^{\prime \prime} \mathrm{S}, 48^{\circ} 19^{\prime} 21^{\prime \prime} \mathrm{W}$ \\
\hline & & $10^{\circ} 13^{\prime} 18^{\prime \prime} \mathrm{S}, 48^{\circ} 21^{\prime} 51^{\prime \prime} \mathrm{W}$ \\
\hline & & $10^{\circ} 07^{\prime} 49^{\prime \prime} \mathrm{S}, 48^{\circ} 18^{\prime} 25^{\prime \prime} \mathrm{W}$ \\
\hline & \multirow{3}{*}{ Porto Nacional } & $10^{\circ} 41^{\prime} 54^{\prime \prime} \mathrm{S}, 48^{\circ} 24^{\prime} 39^{\prime \prime} \mathrm{W}$ \\
\hline & & $10^{\circ} 44^{\prime} 39^{\prime \prime} \mathrm{S}, 48^{\circ} 26^{\prime} 06^{\prime \prime} \mathrm{W}$ \\
\hline & & $10^{\circ} 46^{\prime} 04^{\prime \prime} \mathrm{S}, 48^{\circ} 24^{\prime} 50^{\prime \prime} \mathrm{W}$ \\
\hline & \multirow{2}{*}{ Lagoa da Confusão } & $10^{\circ} 47^{\prime} 54^{\prime \prime} \mathrm{S}, 49^{\circ} 37^{\prime} 21^{\prime \prime} \mathrm{W}$ \\
\hline & & $10^{\circ} 43^{\prime} 50^{\prime \prime} \mathrm{S}, 49^{\circ} 32^{\prime} 27^{\prime \prime} \mathrm{W}$ \\
\hline \multirow{23}{*}{ Roraima } & Boa Vista & $02^{\circ} 48^{\prime} 30^{\prime \prime} \mathrm{N}, 60^{\circ} 44^{\prime} 27^{\prime \prime} \mathrm{W}$ \\
\hline & Anauá Park & $02^{\circ} 49^{\prime} 52^{\prime \prime} \mathrm{N}, 60^{\circ} 40^{\prime} 47^{\prime \prime} \mathrm{W}$ \\
\hline & Caracaraí & $01^{\circ} 25^{\prime} 16^{\prime \prime} \mathrm{N}, 60^{\circ} 59^{\prime} 17^{\prime \prime} \mathrm{W}$ \\
\hline & Estação Ecológica de Viruá & $01^{\circ} 29^{\prime} 24^{\prime \prime} \mathrm{N}, 61^{\circ} 00^{\prime} 07^{\prime \prime} \mathrm{W}$ \\
\hline & Maloca da Raposa & $03^{\circ} 50^{\prime} 25^{\prime \prime} \mathrm{N}, 59^{\circ} 58^{\prime} 24^{\prime \prime} \mathrm{W}$ \\
\hline & Mucajaí & $02^{\circ} 41^{\prime} 05^{\prime} \mathrm{N}, 61^{\circ} 12^{\prime} 56^{\prime \prime} \mathrm{W}$ \\
\hline & Villa Villena & $02^{\circ} 12^{\prime} 30^{\prime \prime} \mathrm{N}, 60^{\circ} 05^{\prime} 06^{\prime \prime} \mathrm{W}$ \\
\hline & Igarapé do Fogo & $02^{\circ} 18^{\prime} 53^{\prime} \mathrm{N}, 60^{\circ} 04^{\prime} 56^{\prime \prime} \mathrm{W}$ \\
\hline & Taboca & $02^{\circ} 27^{\prime} 25^{\prime \prime} \mathrm{N}, 60^{\circ} 12^{\prime} 32^{\prime \prime} \mathrm{W}$ \\
\hline & Açude do Barroso & $02^{\circ} 30^{\prime} 38^{\prime \prime} \mathrm{N}, 60^{\circ} 17^{\prime} 54^{\prime \prime} \mathrm{W}$ \\
\hline & Tepequém & $03^{\circ} 41^{\prime} 31^{\prime \prime} \mathrm{N}, 61^{\circ} 42^{\prime} 41^{\prime \prime} \mathrm{W}$ \\
\hline & Alto Alegre & $02^{\circ} 53^{\prime} 54^{\prime \prime} \mathrm{N}, 61^{\circ} 29^{\prime} 30^{\prime \prime} \mathrm{W}$ \\
\hline & Caracaranã Lake & $03^{\circ} 46^{\prime} 21^{\prime \prime} \mathrm{N}, 59^{\circ} 50^{\prime} 02^{\prime \prime} \mathrm{W}$ \\
\hline & \multirow{3}{*}{$\begin{array}{c}\text { Estação Ecológica de } \\
\text { Maracá }\end{array}$} & $03^{\circ} 22^{\prime} 51^{\prime \prime} \mathrm{N}, 61^{\circ} 26^{\prime} 13^{\prime \prime} \mathrm{W}$ \\
\hline & & $03^{\circ} 22^{\prime} 13^{\prime \prime} \mathrm{N}, 61^{\circ} 26^{\prime} 13^{\prime \prime} \mathrm{W}$ \\
\hline & & $03^{\circ} 21^{\prime} 46^{\prime \prime} \mathrm{N}, 61^{\circ} 25^{\prime} 59^{\prime \prime} \mathrm{W}$ \\
\hline & Serra do Murupu & $03^{\circ} 08^{\prime} 55^{\prime \prime} \mathrm{N}, 60^{\circ} 40^{\prime} 54^{\prime \prime} \mathrm{W}$ \\
\hline & \multirow{6}{*}{$\begin{array}{l}\text { Localities known only } \\
\text { from the coordinates }\end{array}$} & $04^{\circ} 11^{\prime} 46^{\prime \prime} \mathrm{N}, 60^{\circ} 47^{\prime} 56^{\prime \prime} \mathrm{W}$ \\
\hline & & $03^{\circ} 51^{\prime} 42^{\prime \prime} \mathrm{N}, 60^{\circ} 12^{\prime} 58^{\prime \prime} \mathrm{W}$ \\
\hline & & $02^{\circ} 55^{\prime} 37^{\prime \prime} \mathrm{N}, 60^{\circ} 42^{\prime} 57^{\prime \prime} \mathrm{W}$ \\
\hline & & $03^{\circ} 05^{\prime} 18^{\prime \prime} \mathrm{N}, 60^{\circ} 52^{\prime} 52^{\prime \prime} \mathrm{W}$ \\
\hline & & $02^{\circ} 14^{\prime} 38^{\prime \prime} \mathrm{N}, 60^{\circ} 06^{\prime} 13^{\prime \prime} \mathrm{W}$ \\
\hline & & $02^{\circ} 16^{\prime} 58^{\prime \prime} \mathrm{N}, 60^{\circ} 05^{\prime} 16^{\prime \prime} \mathrm{W}$ \\
\hline
\end{tabular}

\subsection{Surveyed sites with Omalonyx spp. absence}

Despite the surveying effort, there were no records of Omalonyx spp. in three States: Distrito Federal (Central-west region), Tocantins and Roraima (North region). The coordinates are shown in Table 5.

\section{Modeling of species distributions}

The potential distribution of each species is indicated in the maps (Figure 2) where different colors and color densities are related to different probabilities of occurrence of suitable conditions for their presence. The environmental model applied here agrees with the data on species distribution (Figure 1) and supports geographic constraints on the species. Some species presents overlapping ranges of distribution. This is the case for $O$. matheroni, O. pattersonae and O. geayi, which tend to occur in tropical areas within the northern half of South America (Figure 2A, B and C) and for $O$. convexus and $O$. unguis, which tend to occur in temperate areas in the southern half of South America (Figure $2 \mathrm{D}$ and E). Omalonyx matheroni, $O$. pattersonae and $O$. geayi present moderate probability of occurrence in a vast area within northern South America, merged with intermittent areas of high probability of occurrence (Figure 2A, B and C). Omalonyx convexus and $O$. unguis present a smaller area of occurrence; however, there are many regions of high probability of occurrence.

There was a high probability of occurrence of $O$. matheroni in the Lesser Antilles, in the central region of South America (hydrographic region 5 and 10), in the Atlantic coast (hydrographic region nine), and in the south of South America (hydrographic region 13) (Figure 2A). These areas are predominantly tropical; however, there are also some temperate areas.

Omalonyx pattersonae showed a high probability of occurrence in tropical and arid regions (Figure 2B) in hydrographic region one, two and three (at isolated points in arid regions within hydrographic regions two and three) and in hydrographic regions six, seven and eight (in tropical and arid regions). Moderate probabilities of occurrence of $O$. pattersonae were observed in hydrographic regions nine and five (tropical region), where discontinuous areas of high probability of occurrence were observed in the Amazonian region (tropical and arid region) (Figure 2B).

Omalonyx geayi is a predominantly tropical species that can also occur in arid areas with high probability of occurrence within hydrographic regions one, two, three, four and five (Figure 2C). In hydrographic region five there are several small areas of high probability of occurrence merged in a vast area of moderate probability. There are areas of moderate probability of occurrence of $O$. geayi in hydrographic regions six, seven and 13 .

Temperate areas along the southeast of South America present the highest probability of $O$. convexus occurrence. Areas with high probability of occurrence are within hydrographic regions 10, 12 and 13 (Figure 2D). There is moderate probability of occurrence in a vast area of hydrographic region 10 and in small areas within hydrographic regions 11 and 13.

Temperate areas in the southeast of South America that presented high probability of $O$. unguis occurrence includes hydrographic regions 10, 11, 12 and 13 (Figure 2E). Some areas within tropical (hydrographic regions five, nine and 10), arid (hydrographic region 11) and temperate areas (hydrographic region 14 and Malvinas Islands) were considered to have moderate probability of occurrence.

The PCA analysis resulted in three layers with highest eigenvalues that better explain the relationship between the variables in the selected study area. The most representative variables within these PCA-analysis axes were BIO4 (Temperature seasonality), BIO12 (Annual precipitation), and Altitude. As a consequence, the modeling of the species of Omalonyx was strongly influenced by these three variables, and their potential distribution was mainly determined by them. There is only one record of O. brasiliensis, thus, it was not possible to model this species' distribution due to technical limitations.

\section{Discussion}

This work addresses Omalonyx species distribution. The genus has a widespread Neotropical distribution, covering most South American hydrographic regions (except hydrographic regions one and 14) and the Lesser Antilles. The species occurrence map and the model of species distribution showed that three species are related to the north of South America (O. matheroni, O. pattersonae, O. geayi) and Lesser Antilles, and three species are related to the south of South America (O. unguis, O. convexus, $O$. brasiliensis).

Omalonyx matheroni is the most widespread species, and its distribution is more concentrated in northern South America (North and Northeast of Brazil, Guyana, French Guiana and Suriname) and in the Lesser Antilles. Omalonyx matheroni occurs in all Brazilian hydrographic regions. The most southern record for this species is Santa Catarina State in the south of Brazil (12, Figure 1).

Among northern species, O. matheroni is the most widespread. On the other hand, the records for $O$. geayi and $O$. pattersonae are discontinuous. 


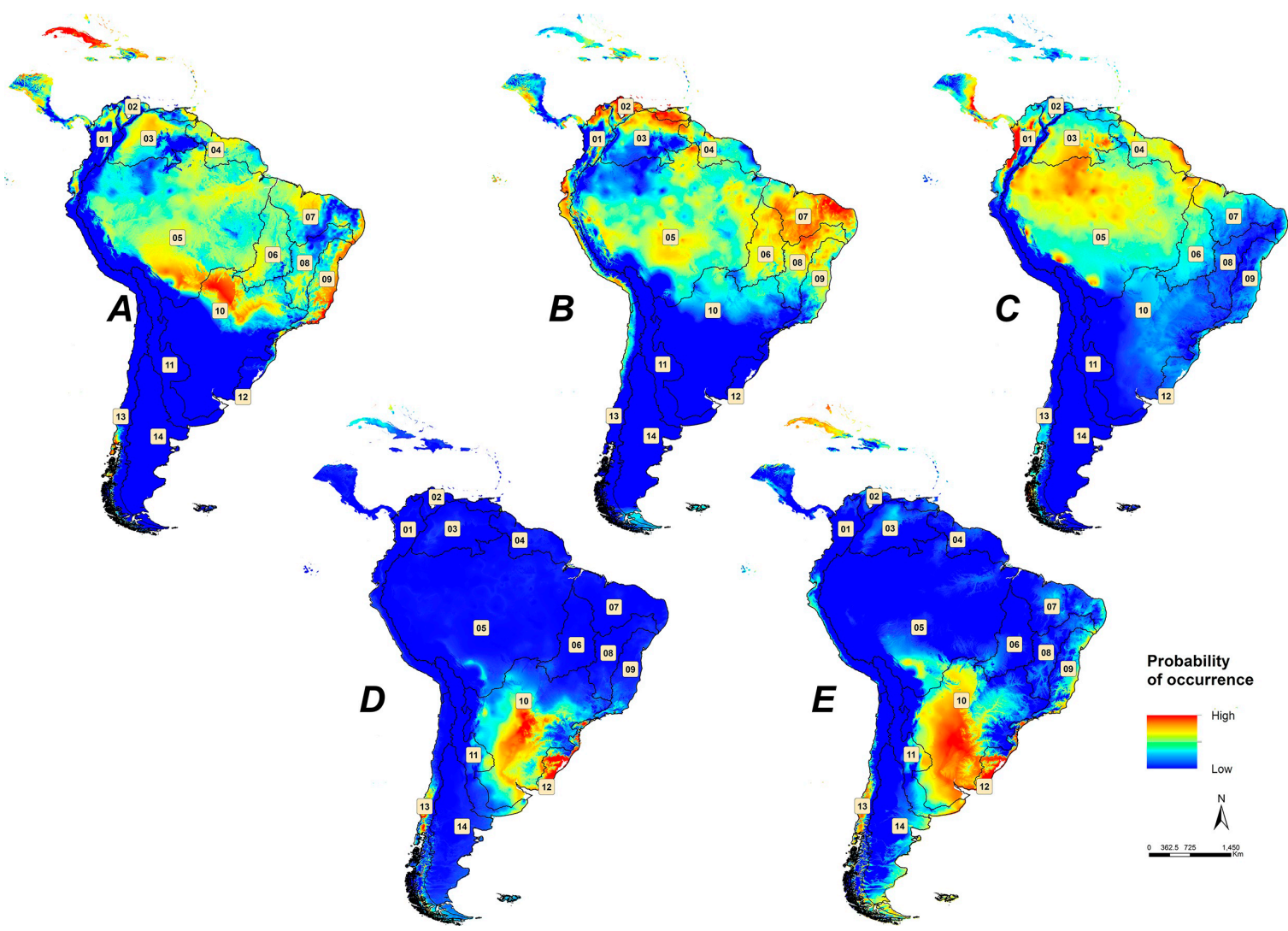

Figure 2 Potential distribution of Omalonyx species modeled with Bioclim analyses using all 19 bioclimatic variables, altitude and the full dataset showed on tables 2 and 3. (A) Omalonyx matheroni; (B) Omalonyx pattersonae, (C) Omalonyx geayi, (D) Omalonyx convexus, (E) Omalonyx unguis. The numbers represents the mainwatersheds within South America. The hydrographic regions are indicated by numbers: 1) Pacific Coast; 2) Caribbean sea; 3) Orinoco River; 4) Amapá-Esequibo; 5) Amazon River; 6) Tocantins River; 7) North Atlantic; 8) São Francisco River; 9) Eastern Atlantic; 10) Del Plata; 11) Border strip of the Brasilica (North) and the Chilean-Patagonian (South West) Subregion; 12) Eastern; 13) Chilean-Patagonian Subregion of the Pacific Versant; 14) Chilean-Patagonian Subregion of the Atlantic Versant.

Despite a huge area with high or intermediate probability of occurrence (Figure 2), the records for O. pattersonae and O. geayi are sparser (Figure 1). Omalonyx geayi was known to occur only in Kaw Swamp, French Guiana, the type locality (Tillier 1981). However, new records published recently expanded its distribution to Ecuador, Bolivia, Suriname and some localities in Brazil (Arruda et al. 2016). In this work, we recorded O. pattersonae for the first time in three hydrographic regions in North and Northeast of Brazil. There is one state in Brazil (Amazon State) where all three of these species occur at sites that are very close.

The other three species (O. unguis, O. convexus, O. brasiliensis) are concentrated in the south of South America. Among these species O. unguis reaches the most northern positions. This species was even found outside its distribution range (Lesser Antilles, Bolivia, Peru and in Ceará State, in the Northeast of Brazil). Omalonyx convexus records are very concentrated (Figure 1) and are restricted to sites where high or intermediate probability of occurrence was predicted (Figure 2B). However, there are some exceptions, and the species was also recorded in the north shore of Venezuela and in the Northeast of Brazil (Sergipe State). Until now, O. brasiliensis was known only from its type locality in Rio Grande do Sul State, Brazil. Despite all the surveying efforts already concentrated in Rio Grande do Sul State (Figure 1), O. brasiliensis was never found again since its description and the species remain known only for the type specimens from an unspecified site that could be related to hydrographic region 10 or 12 (Figure 1). Thus, O. brasiliensis remains to be rediscovered. This is a large State where $O$. convexus is widespread (Table 2, Figure 1); thus, both species may occur in sympatry.
There are several records from literature review, museums and collections that were not identified to species level and are designated Omalonyx sp. They were not used to model species distribution. These records are widespread throughout South American hydrographic regions (e.g., five, seven, nine and 10) and should also be carefully analyzed in future studies. On the other hand, some records identified to species level seem to be inconsistent (e.g., O. convexus was recorded for the Northeast of Brazil) and should be revised. All the records situated outside the area predicted for the species distribution should be carefully investigated. We suppose that the lack of taxonomists dealing with this group is the main cause for the great amount of records identified to genus level. On the other hand, the lack of training and the subtle morphological differences among species must be a cause of misidentification. These problems contribute to gaps in the knowledge regarding Omalonyx distribution (Tillier 1981, Coscarelli \& Vidigal 2011).

Brazil is the best-sampled region in South America. Here, based on our malacological surveys, new records were made in Acre, Rondônia, Piaui, and Amapa. However, despite the absence of records even after intensive surveys in some administrative unities (Roraima, Tocantins and Distrito Federal) (Table 5), the genus was registered in all Brazilian hydrographic regions. There were no records for the central area of Tocantins River hydrographic region (surveyed sites: Palmas and other surrounding areas such as Lagoa da Confusão and Porto Nacional). However, the genus was present in the upper part of the hydrographic region (comprising Goiás and Pará States; Figure 1 and Table 2). Distrito Federal is an administrative unity that encompasses a small area when compared to the Brazilian States, 
and within this small area, there are the frontiers of three hydrographic regions: Tocantins River, São Francisco River and Del Plata. Despite its proximity to Goiás State where we found Omalonyx sp., there were no records in Distrito Federal (surveyed sites: the margins of Paranoá Lake, in Brasília, and in Brazlândia, all of them in the upper Del Plata hydrographic region where the genus is widely distributed; Figure 1). In Roraima State, northern Brazil, despite the surveying efforts ( 23 records of absence) no record was made. Roraima is within the Amazon River hydrographic region in which Omalonyx is widely distributed (Figure 2). However, all these negative localities are in the northeastern quadrant of Roraima, which is characterized by unusual vegetation, a seasonally flooded savannah, locally called "Lavrado" (Barbosa et al. 2007), which may have some ecological features that prevent Omalonyx spp. colonization. Future sampling efforts should focus on areas within these States that were not investigated until now. Considering that Omalonyx spp. occur in the hydrographic regions that cover these States, the absence of records is probably due to insufficient sampling effort.

In South America Omalonyx species were found in all hydrographic regions (except hydrographic regions one and 14) and countries. However, there were only isolated records in Colombia, Ecuador and Chile, indicating the need for more sampling effort in these areas (Figure 1). However, the occurrence of this genus in Chile must be further investigated due to different point of views regarding the identification of the Chilean slug-like succineids (Tillier 1981). The record from Chile (Letellier et al. 2014) should be carefully investigated since Tillier (1981) considered that the species recorded in Chile, O. gayana, belongs to the genus Succinea. In fact, Chile is separated from the other South American countries by the Andes mountain range, which is the major water divider in South America. d'Orbigny (1837) stated that the genus occurs only on the east side of the ridge, and until now, there is no evidence suggesting otherwise. Our results agree with those from d'Orbigny (1837) and Tillier (1981). Bioclimatic analysis proved to be a valuable tool for gaining insight into the geographical distribution of a species, especially when few records are available (i.e., $O$. geayi and $O$. pattersonae). Predictions showed that $O$. matheroni and $O$. geayi are tropical species. Omalonyx pattersonae usually occurs in arid regions; however, its distribution moderately extends over tropical areas. However, the prediction of $O$. geayi to hydrographic region 13 (temperate and very cold area) could be related to low numbers of records. In fact, bias can occur if species are insufficiently sampled. Thus, climatic envelopes may generate incomplete results, and the accuracy of predicted distributions will be decreased (Beaumont et al. 2005).

The occurrence records and the predictions both indicated that $O$. convexus and $O$. unguis have largely overlapping distributions in temperate areas within southern South America. These results are in accordance with those from Arruda \& Thomé (2008b) and Coscarelli \& Vidigal (2011) However, the distribution of $O$. convexus is constrained to temperate regions while $O$. unguis is well represented in tropical, temperate and arid regions indicating that the latter presents a broader tolerance range for environmental variables and is adapted to different bioclimatic conditions.

Considering the extension and the great diversity of habitats in the neotropical region and the wide occurrence area of Omalonyx species, bioclimatic models can be used to extrapolate information on species distribution if the high cost of field surveys in such a vast area is unfeasible. Information on the distribution of Omalonyx species will help to improve the taxonomic and biogeographic studies on these slug-like gastropods.

\section{Acknowledgements}

We are grateful to all colleagues that collected and sent material from different localities: Silvana Thiengo, Mônica Fernandez, Jean P. Pointier, Andrea Roche, Diego E. Gutierrez, Fernando S. Bezerra, José Cassimiro, Henry P. Lima, Felipe S. F. Leite and Villand Bergerhoff. We are also grateful to the curators of the institutional collections Célio Magalhães (INPA), Luiz R. Simone (MZSP), Arnaldo Coelho and Norma Salgado (MNRJ), Alejandra Rumi (MLP). Special thanks to Sílvia Gandolfi and Sônia Buck for the valuable support, to Daniel M. Pimpão, Fernando I. Martins, Linus Spatz and Mônica Tassara for important assistance in field collections, Adalberto dos Santos and Jennifer T. M. Andrade for text review. This work was partially supported by grants from FAPEMIG.

\section{Author Contributions}

Ms. Daniel Coscarelli: contributed to the conception and design of the study. He did most of the malacological surveys, dissections (morphological identification) and visits to museums and collections. Moreover he wrote the first version of the manuscript and after some changes in the manuscript structure, he critically reviewed the paper.

Dra. Lângia C. Montresor: contributed to this manuscript since the beginning of the project. She reviewed the project that was submitted in order to get grants. She visited malacologycal collections in Rio de Janeiro State and also provided some field specimens. She is working in the manuscript since the first versions, writing and critically revising the text and improving the quality of the analysis.

Dr. Alan de Melo: has a vast experience with invertebrates and scientific writing and improved the quality of the manuscript. The contributed to data analysis and interpretation and critically revised the manuscript.

Philipp Russo: used our distribution data and WorldClim database to build the models of species distribution using the Maximum Entropy Algorithm (MaxEnt).

Teofânia Vidigal: contributed to the conception and design of the study. She wrote the project and got the grants that gave origin to this paper. She supervised and coordinated the project. She also provided field specimens, dissected (morphological identification), analyzed data and worked in the manuscript since the first until the last version, critically revising the manuscript.

\section{Conflicts of interest}

The authors declare that they have no conflict of interest related to the publication of this manuscript.

\section{References}

AGUDO-PADRON, A.I. 2008. Listagem sistemática dos moluscos continentais ocorrentes no Estado de Santa Catarina, Brasil. Comun. Soc. Malacol. Urug. 9(91):147-179.

AGUDO-PADRON, A.I. 2012. Nuevos aportes a la lista sistemática de moluscos continentales ocurrentes en el Estado de Santa Catarina, Brasil. Amici Molluscarum. 20(1):35-42.

ARAÚJO, M.B. \& RAHBEK, C. 2006. How does climate change affect biodiversity? Science. 313:1396-1397.

ARRUDA, J.O., BARKER, G.M. \& THOMÉ, J.W. 2016. Revaluation of the taxonomic characters and distribution of Omalonyx geayi Gastropoda, Succineidae). Iheringia, Sér. Zool. 106:2016019.

ARRUDA, J.O., GOMES, S.R., RAMÍREZ, R. \& THOMÉ, J.W. 2006. Morfoanatomia de duas espécies do gênero Omalonyx (Mollusca, Pulmonata, Succineidae) com novo registro para Minas Gerais, Brasil. Biociências. 14:61-70.

ARRUDA, J.O. \& THOMÉ, J.W. 2008a. Synonymization of Neohyalimax, Simroth, 1896 and Omalonyx d'Orbigny, 1837, with redescription of Omalonyx brasiliensis (Simroth, 1896) (Gastropoda: Succineidae). The Nautilus 122:96-98.

ARRUDA, J.O. \& THOMÉ, J.W. 2008b. Revalidation of Omalonyx convexus (Hynemann 1868) and emendation of the type locality of Omalonyx unguis (Orbigny 1837). Arch. Moll. 137:159-166. 
ARRUDA, J.O. \& THOMÉ, J.W. 2011. Biological aspects of Omalonyx convexus (Mollusca, Gastropoda, Succineidae) from the Rio Grande do Sul State, Brazil. Biotemas. 24 (4):95-101.

ARRUDA, J.O., PEREIRA, D., BERGONCI, P.E.A., SANTOS, C.P. \& MANSUR, M.C.D. 2009. Novos registros de Omalonyx matheroni (Potiez \& Michaud, 1835) (Mollusca: Gastropoda: Succineidae) para os Estados de São Paulo e Paraná, Brasil. Biotemas. 22:187-190.

BAKER, H.B. 1925. The Mollusca collected by the University of Michigan-Williamson Expedition in Venezuela, Part III: Pupillidae to Oleacinidae. Occas. Pap. Mus. Zool. Univ. Mich. 156:1-56.

BAKER, H.B. 1926. The Mollusca collected by the University of Michigan-Williamson Expedition in Venezuela, Part IV. Occas. Pap. Mus. Zool. Univ. Mich. 157:1-65.

BARBOSA, R.I., CAMPOS, C., PINTO, F. \& FEARNSIDE, P.M. 2007. The "Lavrados" of Roraima: Biodiversity and conservation of Brazil's Amazonian Savannas. Func. Ecosyst. Commun. 1:29-41.

BARKER, M.G. 2001. Gastropods on land: phylogeny, diversity and adaptive morphology. In The biology of terrestrial mollusks (M.G. Barker, ed.). CABI Publishing, New York, USA, p. 1-146.

BEAUMONT, L.J., HUGHES, L. \& POULSEN, M. 2005. Predicting species distributions: use of climatic parameters in BIOCLIM and its impact on predictions of species' current and future distributions. Ecol. Model. 186:250-269.

BONETTO, A.A. 1994. Austral rivers of South America. In Limnology now: a paradigm of planetary problems (Margaleff R. ed.). Elsevier Science, Amsterdam, n. 17 , p. $425-472$.

BRADIE, J. \& LEUNG, B. 2017. A quantitative synthesis of the importance of variables used in MaxEnt species distribution models. J. Biogeogr. 44:1344-1361.

CANTANHEDE, S.P.D., FERNANDEZ, M.A., MATTOS, A.C., MONTRESOR, L.C., SILVA-SOUZA, N. \& THIENGO, S.C. 2014. Freshwater gastropods of the Baixada Maranhense Microregion, an endemic area for schistosomiasis in the State of Maranhão, Brazil: I - qualitative study. Rev. Soc. Bras. Med. Trop. 47:79-85.

CAZZANINGA, N.J. 1985. Anotaciones sobre algunos gasterópodos no marinos de la Argentina. Comun. Soc. Malacol. Urug. 6:329-331.

COSCARELLI, D. \& VIDIGAL, T.H.D.A. 2011. Mollusca, Gastropoda, Succineidae, Omalonyx unguis (d'Orbigny, 1835): Distribution extension and new records for Brazil. Check List. 7:400-403.

COSTA, G.C., NOGUEIRA. C., MACHADO, R.B. \& COLLI, G.R. 2010. Sampling bias and the use of ecological niche modeling in conservation planning: a field evaluation in a biodiversity hotspot. Biodivers. Conserv. 19:883-899.

D'ORBIGNY, A. 1835a. Synopsis terrestrium et fluviatilum molluscorum, in suo per americam meridionalem itinere, ab A. d'Orbigny, collectorum. Mag. Zool. 5:1-44.

D’ORBIGNY, A. 1835b. Voyage dans L'Amerique Méridionale: Mollusques. v.5, Partie 3 - Planches. P. Bertrand, Paris, France.

D’ORBIGNY, A. 1837. Voyage dans L'Amerique Méridionale: Mollusques. v.5, Partie 3. P. Bertrand, Paris, France.

DUTRA-CLARKE, A.V.C., WILLIAMS, C., DICKSTEIN, R., KAUFER, N. \& SPOTILA, J.R. 2001. Inferences on the phylogenetic relationships of Succineidae (Mollusca, Pulmonata) based on 18S rRNA gene. Malacologia. 43:223-236.

EDUARDO, J.M., TAKAHASHI, F.Y., HOLCMAN, M.M., DA COSTA, C.B.T.L. \& OHLWEILER, F. 2012. Freshwater gastropods and helminthes associated, in water collections from Santo André, São Paulo, Brazil. Biociências. 18:22-34.

ELITH, J., PHILliPS, S.J., HASTIE, T., DUDÍK, M., CHEE, Y.E. \& YATES, C.J. 2011. A statistical explanation of MaxEnt for ecologists. Divers. Distrib. 17(1):43-57.

ESCARBASSIERE, R.M. 1993. Nota acerca de la presencia de la babosa Omalonyx (O.) pattersonae Tillier, 1981 (Gastropoda-Pulmonata Succineidae) en Venezuela. Acta Biol. Venez. 14:65-69.

FRANCESCHINI, M.C., POI DE NEIFF, A. \& GALASSI M.E. 2010. Is the biomass of water hyacinth lost through herbivory in native areas important? Aquat. Bot. 92:250-256.

GARCIA, M.V.B., ARRUdA, J.O., PIMPÃO, D.M. \& GARCIA, T.B. 2012. Ocorrência e controle de lesmas do gênero Omalonyx (Gastropoda, Succineidae), pragas de capim-elefante Pennisetum purpureum (Poaceae) em Rio Preto da Eva, Amazonas. Acta Amaz. 42(2):227-230.
GIBBONS, J.S. 1879. Comparison of Omalonyx unguis, D’Orb. with O. felina, Guppy. J. Conchol. 2:99-101.

GUTIÉRREZ GREGORIC, D.E., NÚÑEZ, V., VOGLER, R.E., BELTRAMINO, A.A. \& RUMI, A. 2013 Gasterópodos terrestres de la provincia de Misiones, Argentina. Rev. Biol. Trop. 61(4):1759-1768.

HAAS, F. 1939. Zur Kenntnis der Binnen-Mollusken NO - Brasiliens. Senckenb. Biol. 21:254-278.

HERMANN, P.W. \& DUNDEE, D.S. 1967. Notes on Omalonyx. Sterkiana. 28:1-6.

HERRMANNSEN, N.A. 1849. Indicis generum malacozoorum primorida. v.2. Theodore Fischer, Cassells, Ortmann, Berlin.

HEYNEMANN, F.D. 1868. Die Mundtheile einiger brasilianischen Land- und Süsswasserschnecken. Malakozool. Blätter 15:99-113.

HIDALGO, J.G. 1869. Moluscos del viaje al pacífico verificado de 1862 á 1865 por una comisión de naturalistas enviada por el gobierno español. Parte primera, Univalvos terrestres. Imprenta de Miguel Ginesta, Madrid, Spain.

HIDALGO, J.G. 1870. Catalogue des coquilles terrestres recueillies par naturalistes de la commission scientifique espagnole sur divers points de l'Amérique méridionale. J. Conchol. 18:27-70.

HIDALGO, J.G. 1872. Moluscos del viaje al pacífico verificado de 1862 a 1865 por uma comision de naturalistas enviada por el gobierno español. Parte primera, Univalvol terrestres. Madrid: Imprenta de Miguel Ginesta.

HYLTON-SCOTT, M.I. 1971. Homalonyx weyrauchi, Nueva especie de Tucuman. Neotropica. 17:12-14.

HYLTON-SCOTT, M.I. \& LAPUENTE, E. 1968. Valor diagnostico de la Radula para especies del genero Omalonyx Orbigny. Neotropica 14:49-56.

JESUS, L.S. \& MANSO, C. L.C. 2010. Inventário da coleção de referência de moluscos terrestres e límnicos do LABIMAR, Campus Prof. Alberto Carvalho da Universidade Federal de Sergipe. Scientia Plena. 6(12):121001.

LANGE DE MORRETES, F. 1949. Ensaio de Catálogo dos Moluscos do Brasil. Arq. Mus. Para. 7:1-216.

LETELIER, S.V., BAEZ, P.R., REBOLlEDO, A.U. \& FABRES, A. C. 2014. Moluscos Terrestres Nativos y Exóticos del Archipiélago Juan Fernández (AJF) (Mollusca: Gastropoda). In Boletín del Museo Nacional de Historia Natural, Chile (Herman Núñez C. ed.). Editora Museo Nacional de Historia Natural Casilla, Santiago de Chile, n. 63, p.187-199.

LESSON, M. 1838. Description d'une espèce nouvelle du genre Testacelle et synopsis d'une monographie de ce genre de Mollusques. Rev. Bras. Zool. 1:249-251.

LUTZ, A. 1921. Obervações sobre o gênero Urogonimus e uma nova forma de Leucochloridium em novo hospedador. Mem. Inst. Oswaldo Cruz. 13:136-140.

MALTCHIK, L., STENERT, C. KOTZIAN, C.B. \& PEREIRA, D. 2010. Responses of freshwater molluscs to environmental factors in Southern Brazil wetlands. Braz. J. Biol. 70(3):473-482.

MARTENS, E.V. 1868. Ueber südbrasilische Land und Süsswasser mollusken. Malakozool. Blätter. 15:169-217.

MARTENS, E.V. 1873. Die Binnenmollusken Venezuela's. In Festschrift zur Feier des hundertjahrigen Bestehens der Gesellschaft naturforschender Freunde zu Berlin (Ferd. D. Verlagsbuchhandlung ed.). Harrwitz \& Grossmann, Berlin, Germany, p.157-225.

MARTINEZ, R.E. 1993. Nota acerca de la presencia de la babosa Omalonyx (O.) pattersonae Tillier, 1981 (Gastropoda-Pulmonata: Succineidae) en Venezuela. Acta Biol. Venez. 14:65-69.

MONTRESOR, L.C., VIDIGAL, T.H.D.A., MENDONÇA, C.L.G.F., FERNANDES, A.A., DE SOUZA, K.N., CARVALHO, O.S., CAPUTO, L.F.G., MOTA, E.M. \& LENZI H.L. 2008. Angiostrongylus costaricensis (Nematoda: Protostrongylidae): migration rote in experimental infection of Omalonyx sp. (Gastropoda: Succineidae). Paras. Res. 103:1339-1346.

MORICAND, S. 1836. Troisième supplément au mémoire sur les coquilles terrestres et fluviates de la Province de Bahia. Sociétéde Physique et d'Historie naturelle de Genève, Genébra, 7:415-446.

MOZZER, L.R., MONTRESOR, L.C., VIDIGAL, T.H.D.A. \& LIMA, W.S. 2011. Angiostrongylus vasorum: Experimental infection and larval development in Omalonyx matheroni. J. Parasitol. Res. 2011:1-4 
ODHNER, N.H. 1922. Mollusca from Juan Fernandez and Easter Island. In The Natural History of Juan Fernandez and Easter Island (C. Skottsberg ed.). Zoology. Almqvist \& Wiksells, Uppsala, v.3, p.219-254.

OLAZARRI, J. 1979. Los Moluscos plaga de los cultivos de "berro" en Salto, Uruguay. Comun. Soc. Malacol. Urug. 5: 63-69.

OLIVEIRA, M.P. \& ALMEIDA, M.N. 2000. Inventário preliminar dos Moluscos do Estado de Minas Gerais, Brasil. Strombus. 6:01-06.

PARODIZ, J.J. 1957. Catalogue of the land Mollusca of Argentina. The Nautilus. 70:127-135.

PARODIZ, J.J. 1963. Observaciones anatomicas sobre Omalonyx patera Doer., com una nota biografica acerca de Adolfo Doering (1848-1926). Sterkiana. 12:1-7.

PATTERSON, C.M. 1971. Taxonomic studies of the land snail family Succineidae. Malacol. Rev. 4:131-202.

PEEL, M.C., FINLAYSON, B.L. \& MCMAHON, T.A. 2007. Updated world map of the Koppen-Geiger climate classification. Hydrol. Earth Syst. Sci. Discuss. 4:439-473.

PHILLIPS, S.J., DUDIK, M. \& SCHAPIRE, R.E. 2004. A maximum entropy approach to species distribution modeling. In Proceedings of the Twenty-First International Conference on Machine Learning (C. Brodley coord.). Banff, Alberta, Canada, p. 655-662.

PIMPÃO, D.M. 2007. Moluscos. In Biodiversidade do médio rio Madeira: bases científicas para propostas de conservação (L.H. Rapp Py-Daniel, C.P. Deus, A.L. Henriques, D.M. Pimpão, O.M. Ribeiro eds). INPA/MMA/MCT Manaus, Brazil, p. 69-81

POI DE NEIFF, A., NEIFF, J.J. \& BONETTO, A. 1977. Enemigos naturales de Eichhornia crassipes en el nordeste argentino y posibilidades de su aplicación el control biológico. Ecosur. 4:137-156.

POTIEZ, V.L.V. \& MICHAUD, A.L.G. 1835. Galerie des Mollusques, ou catalogue méthodique, descriptif et raisonné, de mollusques et coquilles du muséum de Douai. J.B. Baillière, Paris, France.

RAMIRES, R. 1991. Primer registro de los géneros Adelopoma Doering, 1884, Ceciloides Férussac, 1814, Pupisoma Stoliczka, 1873 y Omalonyx D’Òrbigny, 1841 (Mollusca, Gastropoda) para el Perú. Puclicaciones Del Museo de Historia Natural Unmsm A, Lima, Perú, n.41, p. 5

RAMÍREZ, R., PAREDES, C. \& ARENAS, J. 2003. Moluscos del Peru. Rev. Biol. Trop. 51 (3): 225-284.

ROBINSON, D.G., HOVESTADT, A., FIELDS, A. \& BREURE, A.S.H. 2009. The land Mollusca of Dominica (Lesser Antilles), with notes on some enigmatic or rare species. Zool. Meded. 83:615-650.
RUBIO, G.D. \& ACOSTA, L.E. 2011. Geographical distribution of the spaceweaving spider, Chibchea salta, from northwestern Argentina: New records and bioclimatic modeling. J. Insect Sci. 11:54.

SALGADO N.C. \& COELHO. A.C.S. 2003. Moluscos terrestres do Brasil (Gastrópodes operculados ou não, exclusive Veronicellidae, Milacidae e Limacidae). Rev. Bio. Trop. 51:149-189.

SCARABINO, F. 2003. Lista sistemática de los Gastropoda terrestres vivientes de Uruguay. Comun. Soc. Malacol. Urug. 8:203-214.

SIMONE, L.R.L. 2006. Land and Freshwater molluscs of Brazil. EGB, Fapesp, São Paulo.

SIMROTH, H. 1896. On Neohyalimax brasiliensis, n. gen., n. sp. (allied to Hyalimax), from Brazil. Proc. Malacol. Soc. Lond. 2:39-45.

TILLIER, S. 1980. Gastéropodes terrestres et fluviatiles de Guyane française. Mém. Mus. Natl. Hist. Nat., Ser. A 118:1-188.

TILLIER, S. 1981. South American and Juan Fernandez succineid slugs (Pulmonata). J. Molluscan Stud. 47:125-146.

TIETZE, E. \& DE FRANCESCO, C. G. 2012. Compositional fidelity of subfossil mollusk assemblages in streams and lakes of the southeastern pampas, Argentina. PALAIOS. 27 (6):401-413.

TRAVASSOS, L.1928. Fauna helminthológica de Mato Grosso. Trematódeos - Parte I. Mem. Inst. Oswaldo Cruz 21:309-741.

VERA-ARDILA, M. L. 2008. Lista de los géneros de moluscos terrestres de Colombia (Mollusca: Gastropoda: Prosobranchia: Mesogastropoda y Pulmonata: Stylommatophora). Biota Colombiana. 9:39-62.

VOGLER, R. E., BELTRAMINO A. A., SEDE, M. M., GUTIÉRREZ GREGORIC, D. E., NÚÑEZ, V. \& RUMI, A. 2013. The giant african snail, Achatina fulica (Gastropoda: Achatinidae): Using bioclimatic models to identify South American areas susceptible to invasion. Am. Malacol. Bull. 31(1):39-50.

ZILLI F, L., MONTALTO, L. \& MARCHESE, M. R 2008. Benthic invertebrate assemblages and functional feeding groups in the Parana' River floodplain (Argentina). Limnologica. 38:159-171.

Received: $24 / 07 / 2017$

Revised: 16/10/2017

Accepted: 23/12/2017

Published online: 01/02/2018 